\title{
An Analog of Leclerc's Conjecture for Bases of Quantum Cluster Algebras
}

\author{
Fan $Q I N$
}

School of Mathematical Sciences, Shanghai Jiao Tong University, Shanghai 200240, People's Republic of China

E-mail: fgin11@sjtu.edu.cn

URL: https://sites.google.com/site/qinfanmath/

Received May 14, 2020, in final form November 13, 2020; Published online November 27, 2020

https://doi.org/10.3842/SIGMA.2020.122

\begin{abstract}
Dual canonical bases are expected to satisfy a certain (double) triangularity property by Leclerc's conjecture. We propose an analogous conjecture for common triangular bases of quantum cluster algebras. We show that a weaker form of the analogous conjecture is true. Our result applies to the dual canonical bases of quantum unipotent subgroups. It also applies to the $t$-analogs of $q$-characters of simple modules of quantum affine algebras.
\end{abstract}

Key words: dual canonical bases; cluster algebras; Leclerc's conjecture

2020 Mathematics Subject Classification: 13F60

\section{Introduction}

\section{$1.1 \quad$ Background}

Dual canonical bases and cluster theory. Let $\mathfrak{g}$ denote a Kac-Moody algebra with a symmetrizable Cartan datum, and $\mathrm{U}_{\mathrm{q}}=\mathrm{U}_{\mathrm{q}}(\mathfrak{g})$ the corresponding quantized enveloping algebra, where $q$ is not a root of unity. The negative (or positive) part $\mathrm{U}_{\mathrm{q}}{ }^{-}$of $\mathrm{U}_{\mathrm{q}}$ possesses the famous canonical bases $[18,19,28,29]$. The corresponding dual basis $\mathrm{B}^{\text {up }}$ also has fascinating properties and is related to the theory of total positivity [30].

Fomin and Zelevinsky invented cluster algebras as a combinatorial framework to understand the total positivity [30] and the dual canonical bases $\mathrm{B}^{\text {up }}$. We refer the reader to the survey [22] for further details of cluster algebras.

Let there be given any Weyl group element $w \in W$. Then the dual canonical basis $\mathrm{B}^{\mathrm{up}}$ of $\mathrm{U}_{\mathrm{q}}{ }^{-}$ restricts to a basis $\mathrm{B}^{\mathrm{up}}(w)=\mathrm{B}^{\mathrm{up}} \cap \mathrm{A}_{\mathrm{q}}\left[N_{-}(w)\right]$ for the quantum unipotent subgroup $\mathrm{A}_{\mathrm{q}}\left[N_{-}(w)\right]$, see [25]. Notice that, if $\mathfrak{g}$ is a finite-dimensional semi-simple Lie algebra, then $\mathrm{A}_{\mathrm{q}}\left[N\left(w_{0}\right)\right]$ agrees with $\mathrm{U}_{\mathrm{q}}{ }^{-}$, where $w_{0}$ denotes the longest element in $W$.

Thanks to previous works (such as $[1,2,9,10,11,12]$ ), it is known that the quantum unipotent subgroup $\mathrm{A}_{\mathrm{q}}\left[N_{-}(w)\right]$ is a (partially compactified) quantum cluster algebra $\overline{\mathcal{A}}_{q}\left(t_{0}\right)$, where the initial seed $t_{0}=t_{0}(\vec{w})$ is constructed using a reduced word $\vec{w}$ of $w$. By Fomin and Zelevinsky [7], the dual canonical basis $\mathrm{B}^{\mathrm{up}}(w)$ is expected to contain all quantum cluster monomials, which was formulated as the quantization conjecture for Kac-Moody cases in [25]. This conjecture has been verified for acyclic cases by [26] based on [16, 32], for symmetric semisimple cases and partially for symmetric Kac-Moody cases by [33], for all symmetric KacMoody cases by [17], and recently, for all symmetrizable Kac-Moody cases by [35].

This paper is a contribution to the Special Issue on Cluster Algebras. The full collection is available at https://www.emis.de/journals/SIGMA/cluster-algebras.html 
Leclerc's conjecture. A basis element $b \in \mathrm{B}^{\mathrm{up}} \subset \mathrm{U}_{\mathrm{q}}^{-}$is said to be real if $b^{2} \in q^{\mathbb{Z}} \mathrm{B}^{\text {up }}$. Leclerc proposed the following conjecture regarding the multiplication by a real element of $\mathrm{B}^{\text {up }}$, which is analogous to Kashiwara crystal graph operator.

Conjecture 1.1 (Leclerc's conjecture [27, Conjecture 1]). Assume that $b_{1}$ is a real element of $\mathrm{B}^{\mathrm{up}}$. Then, for any $b_{2} \in \mathrm{B}^{\mathrm{up}}$ such that $b_{1} b_{2} \notin q^{\mathbb{Z}} \mathrm{B}^{\mathrm{up}}$, the expansion of their product on $\mathrm{B}^{\mathrm{up}}$ takes the form

$$
b_{1} b_{2}=q^{h} b^{\prime}+q^{s} b^{\prime \prime}+\sum_{c \neq b^{\prime}, b^{\prime \prime}} \gamma_{b_{1}, b_{2}}^{c} c
$$

where $b^{\prime} \neq b^{\prime \prime}, h<s \in \mathbb{Z}, \gamma_{b_{1}, b_{2}}^{c} \in q^{h+1} \mathbb{Z}[q] \cap q^{s-1} \mathbb{Z}\left[q^{-1}\right]$.

This conjecture was proved by [17] for symmetric Kac-Moody cases using quiver Hecke algebras [23, 24, 36].

Common triangular bases. For a large class of quantum (upper) cluster algebras (called injective-reachable, see Section 3.3), the author introduced the triangular basis $\mathbf{L}^{t}$ for any chosen seed $t$ in [33]. The basis is characterized by a triangular property with respect to the dominance order on the degrees of its basis elements (Section 3.1), whence the name "triangular". It is unique if it exists, and it can be constructed via Lusztig Lemma for Kazhdan-Lusztig type bases [35, Section 6.1]. Notice that the triangular basis $\mathbf{L}^{t}$ depends on the seed $t$.

In [33, Definition 6.1.1], the author further considered the common triangular basis $\mathbf{L}$, such that it gives rise to the triangular bases $\mathbf{L}^{t}$ for all seeds $t$ and the basis elements have wellbehaved degrees under mutations (Sections 3.2 and 4.1). The common triangular basis, if exists, contains all quantum cluster monomials and verifies the Fock-Goncharov dual basis conjecture $[5,6]$. It turns out the dual canonical bases of $\mathrm{A}_{\mathrm{q}}\left[N_{-}(w)\right]$ give rise to the common triangular bases for the corresponding quantum cluster algebras [20, 33, 35]. Also, the collections of the simple modules in monoidal categorification of cluster algebras also provide examples of the common triangular bases $[3,17,20,33]$. In this view, the common triangular bases suggest a generalization of the dual canonical bases in cluster theory, and their existence also implies the possible existence of monoidal categorifications.

\subsection{Main results}

By [20, 33, 35], after localization and rescaling, the dual canonical basis $\mathrm{B}^{\mathrm{up}}(w)$ agrees with the common triangular basis of the corresponding quantum cluster algebra in the sense of [33]. Correspondingly, we formulate the following analog of Leclerc's conjecture.

Conjecture 1.2 (Conjecture 5.3). Conjecture 1.1 is true if we replace the dual canonical basis by the common triangular basis.

Recall that the quantum cluster monomials provide a subset of the real elements in the dual canonical basis $\mathrm{B}^{\mathrm{up}}(w)$ (we conjecture that all real elements take this form, see Conjecture 5.7). Our first main result is the following weaker form of the analogous conjecture.

Theorem 1.3 (Theorem 5.2). Conjecture 1.2 is true for the real basis elements corresponding to quantum cluster monomials.

Theorem 1.3 implies a triangularity property for the $t$-analogs of $q$-characters of simple modules of quantum affine algebras, see Theorem 5.4. By [21, Corollary 4.12], as a stronger version of Theorem 5.4, Conjecture 1.2 holds true for the quantum cluster algebras in this case, see Remark 5.5.

Our second main result follows as a consequence of Theorem 1.3. 
Theorem 1.4 (Theorem 5.6). If we consider the dual canonical basis $\mathrm{B}^{\mathrm{up}}(w)$ of the quantum unipotent subgroup $\mathrm{A}_{\mathrm{q}}\left[N_{-}(w)\right]$, then Conjecture 1.1 holds true for the real elements corresponding to quantum cluster monomials.

In order to study the analog of Leclerc's conjecture and prove Theorem 1.3, we will consider not only triangularity with respect to degrees but also triangularity with respect to codegrees. Correspondingly, we introduce the notion of double triangular bases (Definition 4.5), such that both the degrees and the codegrees of the basis elements are well-behaved. In fact, the two terms $b^{\prime}, b^{\prime \prime}$ in (the analog of) Leclerc's conjecture are determined by the codegree and the degree respectively, see Lemma 5.1.

As the third main result, we verify that the common triangular basis elements have wellbehaved degrees and codegrees.

Theorem 1.5 (Theorem 4.10). If the common triangular basis exists, then it gives rise to the double triangular basis for any seed. Moreover, it is compatibly copointed.

It is worth remarking that, if the cluster algebra is categorified by a rigid monoidal category, then degrees and codegrees are related to the two different ways of taking the dual objects in the category, see [20].

\subsection{Contents}

In Section 2, we briefly review basic notions in cluster theory needed by this paper.

In Section 3.1, we review notions and techniques introduced and studied by [33, 34] such as dominance orders, (co)degrees and (co)pointed functions. In Section 3.2, we define tropical transformation for codegrees in analogous to that for degrees. In Section 3.3, we review the notion of injective-reachability, and define the sets of distinguished functions $\mathbf{I}^{t}, \mathbf{P}^{t}$ for seeds $t$, and we present some properties of injective-reachability and these distinguished functions.

In Section 4, we define various bases whose degrees or codegrees satisfy certain properties. In particular, we introduce the notion of double triangular bases. We discuss the relation between double triangular bases and (common) triangular bases. We prove that common triangular bases have good properties on their codegrees (Theorem 4.10).

In Section 5, we propose an analog of Leclerc's conjecture for common triangular bases (Conjecture 5.3) and show a weaker form holds true (Theorem 5.2). We discuss its consequences for modules of quantum affine algebras (Theorem 5.4, Remark 5.5). We deduce that the weaker form is satisfied by the dual canonical bases of $\mathrm{A}_{\mathrm{q}}\left[N_{-}(w)\right]$ (Theorem 5.6).

\section{Basics of cluster algebras}

We briefly review notions in cluster theory necessary for this paper following [33, 34, 35]. A reader unfamiliar with cluster theory is referred to [2, 22] for background materials.

Denote $\mathbb{k}=\mathbb{Z}\left[q^{ \pm \frac{1}{2}}\right]=\mathbb{Z}\left[v^{ \pm}\right]$, where $v=q^{\frac{1}{2}}$ is a formal parameter. Define $\mathbf{m}=v^{-1} \mathbb{Z}\left[v^{-1}\right]$. Notice that we have a natural bar involution $\overline{()}$ on $\mathbb{k}$ which sends $v$ to $v^{-1}$. Let ()$^{T}$ denote the matrix transposition and []$_{+}$denote the function $\max (0$,$) .$

\section{$2.1 \quad$ Seeds}

Fix a finite set of vertices $I$ and its partition $I=I_{\mathrm{uf}} \sqcup I_{\mathrm{f}}$ into the unfrozen and frozen vertices.

Let there be given a quantum seed $t=\left(\widetilde{B}(t), \Lambda(t),\left(X_{i}(t)\right)_{i \in I}\right)$, where $X_{i}(t)$ are indeterminates, the integer matrices $\widetilde{B}(t)=\left(b_{i j}(t)\right)_{i \in I, j \in I_{\mathrm{uf}}}$ and $\Lambda(t)=\left(\Lambda_{i j}(t)\right)_{i, j \in I}$ form a compatible pair, i.e. there exists some diagonal matrix $D=\operatorname{diag}\left(d_{k}\right)_{k \in I_{\text {uf }}}$ with strictly positive integer diagonals, such that $\widetilde{B}(t)^{T} \Lambda(t)=\left(\begin{array}{ll}D & 0\end{array}\right) . X_{i}(t)$ are called the $i$-th $X$-variables or quantum cluster 
variables associated to $t, \widetilde{B}(t)$ the $\widetilde{B}$-matrix, $\Lambda(t)$ the $\Lambda$-matrix, and $B(t):=\left(b_{i j}(t)\right)_{i, j \in I_{\mathrm{uf}}}$ the principal part of $\widetilde{B}(t)$ or the $B$-matrix.

Lemma $2.1([2])$.

(1) We have $d_{i} b_{i k}(t)=-d_{k} b_{k i}(t)$ for $i, k \in I_{\mathrm{uf}}$.

(2) The matrix $\widetilde{B}(t)$ is of full rank $\left|I_{\mathrm{uf}}\right|$.

Define the following lattices (of column vectors):

$$
\begin{aligned}
& M^{\circ}(t)=\oplus_{i \in I} \mathbb{Z} f_{i}(t) \simeq \mathbb{Z}^{I}, \\
& N_{\mathrm{uf}}(t)=\oplus_{k \in I_{\mathrm{uf}}} \mathbb{Z} e_{k}(t) \simeq \mathbb{Z}^{I_{\mathrm{uf}}},
\end{aligned}
$$

where $f_{i}(t), e_{k}(t)$ denote the $i$-th and $k$-th unit vectors respectively. Denote

$$
N_{\mathrm{uf}}^{\geq 0}(t)=\oplus_{k \in I_{\mathrm{uf}}} \mathbb{N} e_{k}(t) \simeq \mathbb{N}^{I_{\mathrm{uf}}}
$$

Define the linear map $p^{*}: N_{\mathrm{uf}}(t) \rightarrow M^{\circ}(t)$ such that $p^{*} n=\widetilde{B}(t) n$. Let $\lambda$ denote the bilinear form on $M^{\circ}(t)$ such that

$$
\lambda\left(g, g^{\prime}\right)=g^{T} \Lambda(t) g^{\prime} .
$$

Lemma 2.2. For any $i \in I, k \in I_{\mathrm{uf}}$, we have $\lambda\left(f_{i}(t), p^{*} e_{k}(t)\right)=-\delta_{i k} d_{k}$.

The group algebra of $M^{\circ}(t)$ is the Laurent polynomial ring $\mathbb{k}\left[M^{\circ}(t)\right]:=\mathbb{k}\left[X(t)^{m}\right]_{m \in M^{\circ}(t)}=$ $\mathbb{k}\left[X_{i}(t)^{ \pm}\right]_{i \in I}$ with the usual addition and multiplication $(+, \cdot)$, where we denote the Laurent monomial $X(t)^{m}=\prod_{i \in I} X_{i}(t)^{m_{i}}$ for $m=\sum m_{i} f_{i}(t)$.

The quantum Laurent polynomial ring (also called the quantum torus) $\mathcal{L P}(t)$ associated to $t$ is defined as the commutative algebra $\mathbb{k}\left[M^{\circ}(t)\right]$ further endowed with the twisted product $*$ :

$$
X(t)^{m} * X(t)^{m^{\prime}}=v^{\lambda\left(m, m^{\prime}\right)} X(t)^{m+m^{\prime}} .
$$

By the algebraic structure on $\mathcal{L} \mathcal{P}(t)$, we mean $(+, *)$ unless otherwise specified.

The monomials $X(t)^{m}, m \in \mathbb{N}^{I}$, are called the quantum cluster monomials associated to $t$. The Laurent monomials $X(t)^{m}, m \in \mathbb{N}^{I_{\mathrm{uf}}} \oplus \mathbb{Z}^{I_{\mathrm{f}}}$, are called the localized quantum cluster monomials associated to $t$.

Define the $Y$-variables to be $Y_{k}(t):=X(t)^{p^{*} e_{k}(t)}, k \in I_{\mathrm{uf}}$. Denote $Y(t)^{n}=X(t)^{p^{*} n}$ for $n \in N_{\mathrm{uf}}(t)$.

We also define $\mathcal{F}(t)$ to be the skew field of fractions of $\mathcal{L P}(t)$.

For simplicity, we often omit the symbol $t$ when there is no confusion.

\subsection{Mutations}

For any $k \in I_{\text {uf }}$, we have an operation $\mu_{k}$ called mutation in the direction $k$ which gives us a new seed $t^{\prime}=\mu_{k} t=\left(\widetilde{B}\left(t^{\prime}\right), \Lambda\left(t^{\prime}\right),\left(X_{i}\left(t^{\prime}\right)\right)_{i \in I}\right)$, where $X_{i}^{\prime}:=X_{i}\left(t^{\prime}\right)$ are indeterminates. See [2] for precise definitions of $\widetilde{B}\left(t^{\prime}\right), \Lambda\left(t^{\prime}\right)$. Recall that we have $\mu_{k}^{2} t=t$.

Given any initial seed $t_{0}$, we let $\Delta_{t_{0}}^{+}$denote the set of seeds obtained from $t_{0}$ by iterated mutations. Then we have $\Delta_{t_{0}}^{+}=\Delta_{t}^{+}$if $t \in \Delta_{t_{0}}^{+}$. Throughout this paper, we will always work with seeds from the same set $\Delta^{+}=\Delta_{t_{0}}^{+}$, where the initial seed $t_{0}$ is often omitted for simplicity.

For simplicity, denote $t=\left(\widetilde{B}, \Lambda,\left(X_{i}\right)\right)$ and $t^{\prime}=\left(\widetilde{B}^{\prime}, \Lambda^{\prime},\left(X_{i}^{\prime}\right)\right)$.

Denote $v_{k}=v^{d_{k}}$. Recall that there is an algebra isomorphism $\mu_{k}^{*}: \mathcal{F}\left(t^{\prime}\right) \simeq \mathcal{F}(t)$ called the mutation birational map, such that we have

$$
\mu_{k}^{*}\left(X_{k}^{\prime}\right)=v^{\lambda\left(f_{k}, \sum_{j \in I}\left[-b_{j k}\right]_{+} f_{j}\right)} X_{k}^{-1} *\left(X^{\sum_{j \in I}\left[-b_{j k}\right]_{+} f_{j}}+v_{k}^{-1} X^{\sum_{i \in I}\left[b_{i k}\right]_{+} f_{i}}\right)
$$


and, for $i \neq k$,

$$
\mu_{k}^{*}\left(X_{i}^{\prime}\right)=X_{i}
$$

Notice that we can also write $\mu_{k}^{*}\left(X_{k}^{\prime}\right)=X^{-f_{k}+\sum_{j \in I}\left[-b_{j k}\right]+f_{j}} \cdot\left(1+Y_{k}\right)$. Recall that $\left(\mu_{k}^{*}\right)^{2}$ is an identity.

Let there be given any seed $t^{\prime}=\overleftarrow{\mu}_{t^{\prime}, t} t$, where $\overleftarrow{\mu}_{t^{\prime}, t}=\overleftarrow{\mu}=\mu_{k_{r}} \cdots \mu_{k_{2}} \mu_{k_{1}}$ is a sequence of mutations (read from right to left). We define the mutation birational map $\overleftarrow{\mu}_{t^{\prime}, t}^{*}: \mathcal{F}\left(t^{\prime}\right) \simeq \mathcal{F}(t)$ as the composition $\mu_{k_{1}}^{*} \cdots \mu_{k_{r}}^{*}$. It is known that $\overleftarrow{\mu}_{t^{\prime}, t}^{*}$ is independent of the choice for the mutation sequence $\overleftarrow{\mu}_{t^{\prime}, t}$ from $t$ to $t^{\prime}$. Define a sequence of mutations $\overleftarrow{\mu}_{t, t^{\prime}}=\mu_{k_{1}} \mu_{k_{2}} \cdots \mu_{k_{r}}$ correspondingly which is often denoted by $\left(\overleftarrow{\mu}_{t^{\prime}, t}\right)^{-1}$ for simplicity.

Notice that, if $i \in I_{\mathrm{f}}$, we have $\overleftarrow{\mu}_{t^{\prime}, t}^{*} X_{i}\left(t^{\prime}\right)=X_{i}(t)$ for all $t^{\prime} \in \Delta^{+}$. Correspondingly, we call $X_{i}\left(t^{\prime}\right), i \in I_{\mathrm{f}}, t^{\prime} \in \Delta^{+}$, the frozen variables, and denote them by $X_{i}$ for simplicity. Define the set of frozen factors to be $\mathcal{P}=\left\{X^{m} \mid m \in \mathbb{Z}^{I_{\mathrm{f}}}\right\}$.

\subsection{Cluster algebras}

Let there be given a quantum seed $t \in \Delta^{+}$.

Definition 2.3. The (partially compactified) quantum cluster algebra $\overline{\mathcal{A}}_{q}(t)$ is defined to be the $\mathbb{k}$-subalgebra of $\mathcal{L P}(t)$ generated by the quantum cluster variables $\overleftarrow{\mu}_{t^{\prime}, t^{\prime}}^{*} X_{i}\left(t^{\prime}\right), i \in I, t^{\prime} \in \Delta^{+}$

The (localized) quantum cluster algebra $\mathcal{A}_{q}(t)$ is defined to be the localization of $\overline{\mathcal{A}}_{q}(t)$ at $\mathcal{P}$.

The upper quantum cluster algebra $\mathcal{U}_{q}(t)$ is defined to be $\cap_{t^{\prime} \in \Delta^{+}} \overleftarrow{\mu}_{t^{\prime}, t}^{*} \mathcal{L} \mathcal{P}\left(t^{\prime}\right)$

Recall that we have $\overline{\mathcal{A}}_{q}(t) \subset \mathcal{A}_{q}(t) \subset \mathcal{U}_{q}(t)$. Moreover, for $t, t^{\prime} \in \Delta^{+}$, we have $\overleftarrow{\mu}_{t^{\prime}, t}^{*} \mathcal{U}_{q}\left(t^{\prime}\right)=$ $\mathcal{U}_{q}(t), \overleftarrow{\mu}_{t^{\prime}, t}^{*} \mathcal{A}_{q}\left(t^{\prime}\right)=\mathcal{A}_{q}(t), \overleftarrow{\mu}_{t^{\prime}, t}^{*} \overline{\mathcal{A}}_{q}\left(t^{\prime}\right)=\overline{\mathcal{A}}_{q}(t)$. It is sometimes convenient to forget the symbols $t, t^{\prime}$ by viewing $\overleftarrow{\mu}_{t^{\prime}, t}^{*}$ as an identification.

\section{Dominance orders and pointedness}

In this section, we recall the notions and some basic results concerning dominance orders and pointed functions from [33, 34]. We also describe properties of codegrees and copointed functions in analogous to those of degrees and pointed functions.

\subsection{Dominance orders and pointedness}

Let there be given a quantum seed $t$.

Definition 3.1 (dominance order). We denote $g^{\prime} \preceq_{t} g$ if there exists some $n \in N_{\mathrm{uf}}^{\geq 0}(t)$ such that $g^{\prime}=g+p^{*} n$. In this case, we say $g^{\prime}$ is dominated by $g$, or $g^{\prime}$ is inferior to $g$.

The meanings of symbols $\prec_{t}, \succ_{t}, \succeq_{t}$ are given in the obvious way.

Lemma $3.2([33])$. For any $g, g^{\prime} \in M^{\circ}(t)$, there exist finitely many $g^{\prime \prime}$ such that $g^{\prime \prime} \preceq_{t} g$ and $g^{\prime \prime} \succeq_{t} g^{\prime}$.

Notice that $\mathcal{L P}(t)$ has a subring $\mathbb{k}\left[N_{\mathrm{uf}}^{\geq 0}(t)\right]:=\mathbb{k}\left[Y_{k}(t)\right]_{k \in I_{\mathrm{uf}}}$. Let $\mathbb{k}\left[\widehat{\left.Y_{k}(t)\right]_{k}} \in I_{\mathrm{uf}}\right.$ denote the completion of $\mathbb{k}\left[Y_{k}(t)\right]_{k \in I_{\mathrm{uf}}}$ with respect to the maximal ideal generated by $Y_{k}(t), k \in I_{\mathrm{uf}}$. The formal completion of $\mathcal{L P}(t)$ is defined to be

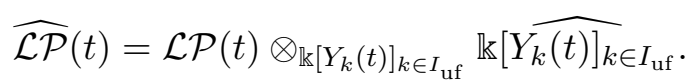

Elements in $\widehat{\mathcal{L P}}(t)$ will be called functions or formal Laurent series. 
Similarly, we consider the subring $\mathbb{k}\left[Y_{k}^{-1}(t)\right]_{k \in I_{\mathrm{uf}}}$ of $\mathcal{L} \mathcal{P}(t)$ and its completion $\mathbb{k}\left[Y_{k}^{-1}(t)\right]_{k \in I_{\mathrm{uf}}}$ with respect to the maximal ideal generated by $Y_{k}^{-1}(t), k \in I_{\mathrm{uf}}$. We define the following completion of $\mathcal{L} \mathcal{P}(t)$ :

$$
\widetilde{\mathcal{L P}}(t):=\mathcal{L P}(t) \otimes_{\mathbb{k}\left[Y_{k}^{-1}(t)\right]_{k \in I_{\mathrm{uf}}}} \mathbb{k}\left[Y_{k}^{-1(t)}\right]_{k \in I_{\mathrm{uf}}} .
$$

By a formal sum, we mean a possibly infinite sum. Let $Z$ denote a formal sum $Z=$ $\sum_{m \in M^{\circ}(t)} b_{m} X(t)^{m}$. Notice that it belongs to $\widehat{\mathcal{L P}}(t)$ (resp. $\left.\widehat{\mathcal{L P}}(t)\right)$ if and only if its Laurent degree support $\operatorname{supp}_{M^{\circ}(t)} Z=\left\{m \mid b_{m} \neq 0\right\}$ has finitely many $\prec_{t}$-maximal elements (resp. finitely many $\prec_{t}$-minimal elements).

Definition 3.3 ((co)degrees and (co)pointedness). The formal sum $Z$ is said to have degree $g$ if $\operatorname{supp}_{M^{\circ}(t)} Z$ has a unique $\prec_{t}$-maximal element $g$, and we denote $\operatorname{deg}^{t} Z=g$. It is said to be pointed at $g$ or $g$-pointed if we further have $b_{g}=1$.

The formal sum $Z$ is said to have codegree $\eta$ if $\operatorname{supp}_{M^{\circ}(t)} Z$ has a unique $\prec_{t^{-m i n i m a l}}$ element $\eta$, and we denote $\operatorname{codeg}^{t} Z=\eta$. It is said to be copointed at $\eta$ or $\eta$-copointed if we further have $b_{\eta}=1$.

Let there be given a set $S$. It is said to be $M^{\circ}(t)$-pointed if it takes the form $S=\left\{S_{g} \mid g \in\right.$ $\left.M^{\circ}(t)\right\}$, where $S_{g}$ are $g$-pointed functions in $\widehat{\mathcal{L P}}(t)$. Similarly, it is said to be $M^{\circ}(t)$-copointed, if it takes the form $S=\left\{S^{\eta} \mid \eta \in M^{\circ}(t)\right\}$, where $S^{\eta}$ are $\eta$-copointed functions in $\overline{\mathcal{L P}}(t)$.

As in [34], if $Z$ is both pointed at $g$ and copointed at $\eta$, it is said to be bipointed at the bidegree $(g, \eta)$.

Definition 3.4 (normalization). Let $\mathcal{F}(\mathbb{k})$ denote the fraction field of $\mathbb{k}$. If $Z$ has degree $g$, we define its (degree) normalization in $\widehat{\mathcal{L P}}(t) \otimes_{\mathbb{k}} \mathcal{F}(\mathbb{k})$ to be

$$
[Z]^{t}:=b_{g}^{-1} Z \text {. }
$$

Similarly, if $Z$ has codegree $\eta$, we define its codegree normalization in $\widetilde{\mathcal{L P}}(t) \otimes_{\mathbb{k}} \mathcal{F}(\mathbb{k})$ to be:

$$
\{Z\}^{t}:=b_{\eta}^{-1} Z \text {. }
$$

Let there be given a (possibly infinite) collection of formal sums $Z_{j}$. Notice that their formal sum $\sum_{j} Z_{j}$ is well-defined if, at each Laurent degrees, only finitely many of them have nonvanishing coefficients.

Definition 3.5 (degree triangularity). A formal sum $\sum_{j} b_{j} Z_{j}$ of pointed elements $Z_{j} \in \widehat{\mathcal{L P}}(t)$, $b_{j} \in \mathbb{k}$, is said to be degree $\prec_{t}$-unitriangular, or $\prec_{t}$-unitriangular for short, if $\left\{\operatorname{deg}^{t} Z_{j} \mid b_{j} \neq 0\right\}$ has a unique $\prec_{t}$-maximal element $\operatorname{deg}^{t} Z_{j_{0}}$ and $b_{j_{0}}=1$. It is further said to be degree $\left(\prec_{t}, \mathbf{m}\right)$ unitriangular, or $\left(\prec_{t}, \mathbf{m}\right)$-unitriangular for short, if we further have $b_{j} \in \mathbf{m}$ for $j \neq j_{0}$.

Definition 3.6 (codegree triangularity). A formal sum $\sum_{j} b_{j} Z_{j}$ of copointed elements $Z_{j} \in$

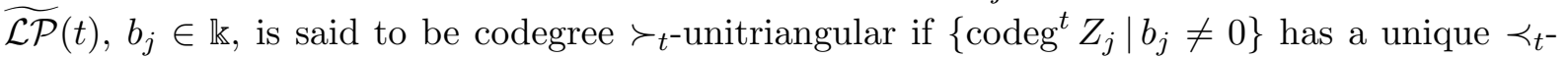
minimal element $\operatorname{codeg}^{t} Z_{j_{0}}$ and $b_{j_{0}}=1$. It is further said to be codegree $\left(\succ_{t}, \mathbf{m}\right)$-unitriangular, if we further have $b_{j} \in \mathbf{m}$ for $j \neq j_{0}$.

Notice that Lemma 3.2 implies that a degree $\prec_{t}$-unitriangular sum is a well-defined sum in $\widehat{\mathcal{L P}}(t)$ and, similarly, a codegree $\succ_{t}$-unitriangular sum is a well-defined sum in $\widehat{\mathcal{L P}}(t)$.

\section{Lemma 3.7.}

(1) Let there be given a $M^{\circ}(t)$-pointed set $S$, then any pointed function $Z \in \widehat{\mathcal{L P}}(t)$ can be written uniquely as a (degree) $\prec_{t}$-unitriangular sum of elements of $S$ [33]. 
(2) Let there be given a $M^{\circ}(t)$-copointed set $S$, then any copointed element $Z \in \widetilde{\mathcal{L P}}(t)$ can be written uniquely as a codegree $\succ_{t}$-unitriangular sum of elements of $S$.

Proof. (1) is proved as in [33, Lemma 3.1.10(i)], see also [34, Definition-Lemma 4.1.1]. (2) can be proved similarly, or we can deduce it from (1) by using the map $\iota$ defined in (3.2).

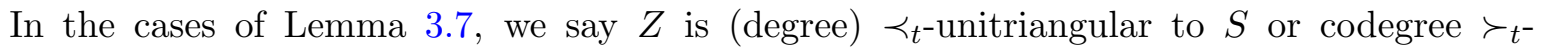
unitriangular to $S$ respectively. It is further said to be (degree) $\left(\prec_{t}, \mathbf{m}\right)$-unitriangular to $S$ or codegree $\left(\succ_{t}, \mathbf{m}\right)$-unitriangular to $S$ respectively, if its decomposition in $S$ has such properties.

Example 3.8 (type $A_{2}$ ). Take $I=\{1,2\}=I_{\mathrm{uf}}$ and $I_{\mathrm{f}}=\varnothing$. Consider the initial quantum seed $t_{0}$ such that $B\left(t_{0}\right):=B:=\left(\begin{array}{cc}0 & -1 \\ 1 & 0\end{array}\right), \Lambda\left(t_{0}\right):=\Lambda:=B$ and denote its quantum cluster variables by $X_{1}, X_{2}$ for simplicity. Then we have $Y_{1}=X^{f_{2}}=X_{2}$ and $Y_{2}=X^{-f_{1}}=X_{1}^{-1}$, where $f_{i}$ denote the $i$-th unit vector.

The corresponding quantum cluster algebra $\mathcal{A}_{q}$ has five quantum cluster variables. It has the basis $\mathbf{L}$ consisting of the quantum cluster monomials.

Recall that, in $\mathcal{L P}\left(t_{0}\right)$, the commutative product is denoted by - or is omitted, while the twisted product is denoted by $*$. The non-initial quantum cluster variables can be written as the following in $\mathcal{L P}\left(t_{0}\right)$ :

$$
\begin{aligned}
& P_{2}:=X^{f_{1}-f_{2}}+X^{-f_{2}}=X^{f_{1}-f_{2}} \cdot\left(1+Y_{2}\right), \\
& I_{2}:=P_{1}:=X^{-f_{2}}+X^{-f_{1}-f_{2}}+X^{-f_{1}}=X^{-f_{2}} \cdot\left(1+Y_{2}+Y_{1} \cdot Y_{2}\right), \\
& I_{1}:=X^{-f_{1}}+X^{-f_{1}+f_{2}}=X^{-f_{1}} \cdot\left(1+Y_{1}\right) .
\end{aligned}
$$

Then $P_{2}, P_{1}, I_{1}$ are bipointed at the bidegrees $\left(f_{1}-f_{2},-f_{2}\right),\left(-f_{2},-f_{1}\right),\left(-f_{1},-f_{1}+f_{2}\right)$ respectively.

Let us compute some degree normalized products in $\mathcal{L P}\left(t_{0}\right)$ :

$$
\begin{aligned}
& {\left[X_{1} * I_{1}\right]=X_{1} * I_{1}=1+v^{-1} X_{2},} \\
& {\left[X_{1} * I_{2}\right]=v^{\Lambda_{12}} X_{1} * I_{2}=X^{f_{1}-f_{2}}+X^{-f_{2}}+v^{-1} \cdot 1=P_{2}+v^{-1} \cdot 1,} \\
& {\left[X_{2} * I_{1}\right]=v^{\Lambda_{21}} X_{2} * I_{1}=X^{f_{2}-f_{1}}+X^{-f_{1}+2 f_{2}},} \\
& {\left[X_{2} * I_{2}\right]=X_{2} * I_{2}=1+v^{-1} X^{-f_{1}}+v^{-1} X^{-f_{1}+f_{2}}=1+v^{-1} I_{1} .}
\end{aligned}
$$

Notice that $v^{\Lambda_{21}} X_{2} * I_{1}$ is a quantum cluster monomial of the seed $\mu_{1}\left(t_{0}\right)$. Then the above normalized products are degree $\left(\prec_{t}, \mathbf{m}\right)$-unitriangular to the basis $\mathbf{L}$.

Similarly, we compute some codegree normalized products in $\mathcal{L P}\left(t_{0}\right)$ :

$$
\begin{aligned}
& \left\{P_{1} * X_{1}\right\}=P_{1} * X_{1}=v^{-\Lambda_{21}} X^{f_{1}-f_{2}}+v^{-\Lambda_{21}} X^{-f_{2}}+1=v^{-1} P_{2}+1, \\
& \left\{P_{1} * X_{2}\right\}=v^{\Lambda_{12}} P_{1} * X_{2}=v^{-1}+X^{-f_{1}}+X^{f_{2}-f_{1}}=v^{-1} \cdot 1+I_{1}, \\
& \left\{P_{2} * X_{1}\right\}=v^{\Lambda_{21}} P_{2} * X_{1}=X^{2 f_{1}-f_{2}}+X^{f_{1}-f_{2}} \\
& \left\{P_{2} * X_{2}\right\}=P_{2} * X_{2}=v^{-1} X_{1}+1
\end{aligned}
$$

Notice that $v^{\Lambda_{21}} P_{2} * X_{1}$ is a quantum cluster monomial of $\mu_{2}\left(t_{0}\right)$. Then the above normalized products are codegree $\left(\succ_{t}, \mathbf{m}\right)$-unitriangular to the basis $\mathbf{L}$.

\subsection{Tropical transformations and compatibility}

As before, let there be given seeds $t^{\prime}=\overleftarrow{\mu} t$, where $\overleftarrow{\mu}=\overleftarrow{\mu}_{t^{\prime}, t}$ is a sequence of mutations. Denote $\overleftarrow{\mu}_{t, t^{\prime}}=\overleftarrow{\mu}_{t^{\prime}, t}^{-1}$. Denote the $i$-th cluster variables associated to $t$ and $t^{\prime}$ by $X_{i}$ and $X_{i}^{\prime}$ respectively. Let $f_{i}, f_{i}^{\prime}$ denote the $i$-th unit vectors associated to $t$ and $t^{\prime}$ respectively. 
Definition 3.9 (tropical transformation). If $t^{\prime}=\mu_{k} t, k \in I_{\mathrm{uf}}$, we define the (degree) tropical transformation $\phi_{t^{\prime}, t}: M^{\circ}(t) \simeq M^{\circ}\left(t^{\prime}\right)$ such that, for any $g=\left(g_{i}\right)_{i \in I} \in M^{\circ}(t) \simeq \mathbb{Z}^{I}$, its image $\phi_{t^{\prime}, t} g=\left(g_{i}^{\prime}\right)_{i \in I} \in M^{\circ}\left(t^{\prime}\right) \simeq \mathbb{Z}^{I}$ is given by

$$
g_{i}^{\prime}= \begin{cases}-g_{k}, & i=k, \\ g_{i}+b_{i k}\left[g_{k}\right]_{+}, & i \neq k, \quad b_{i k} \geq 0 \\ g_{i}+b_{i k}\left[-g_{k}\right]_{+}, & i \neq k, \quad b_{i k}<0\end{cases}
$$

In general, we define the (degree) tropical transformation $\phi_{t^{\prime}, t}: M^{\circ}(t) \simeq M^{\circ}\left(t^{\prime}\right)$ as the composition of the tropical transformations for adjacent seeds along the mutation sequence $\overleftarrow{\mu}$ from $t$ to $t^{\prime}$. By [13], $\phi_{t^{\prime}, t}$ is the tropicalization of certain birational maps between the split algebraic tori associate to $t, t^{\prime}$ and, consequently, independent of the choice of $\overleftarrow{\mu}$.

Recall that $\overleftarrow{\mu}_{t, t^{\prime}}^{*} X_{i}$ is a pointed Laurent polynomial in $\mathcal{L P}\left(t^{\prime}\right)$ by $[4,14,37]$

Definition 3.10 (degree linear transformation [34, Definition 3.3.1]). Define $\psi_{t^{\prime}, t}: M^{\circ}(t) \simeq$ $M^{\circ}\left(t^{\prime}\right)$ to be the linear map such that $\psi_{t^{\prime}, t}\left(f_{i}\right)=\operatorname{deg}^{t^{\prime}} \overleftarrow{\mu}_{t, t^{\prime}}^{*} X_{i}$

By [34, Lemma 3.3.4], the mutation map $\overleftarrow{\mu}_{t, t^{\prime}}^{*}: \mathcal{F}(t) \simeq \mathcal{F}\left(t^{\prime}\right)$ induces an injective algebra homomorphism $\widehat{\mu}: \mathcal{L P}(t) \hookrightarrow \widehat{\mathcal{L P}}\left(t^{\prime}\right)$. It has the following property.

Lemma 3.11 ([34, Lemma 3.3.7]). For any $m \in \mathbb{Z}^{I}, \widehat{\mu} X^{m}$ is a well-defined function in $\widehat{\mathcal{L P}}\left(t^{\prime}\right)$ pointed at degree $\psi_{t^{\prime}, t} m$.

Moreover, for $Z \in \mathcal{L} \mathcal{P}(t) \cap \overleftarrow{\mu}_{t^{\prime}, t}^{*} \mathcal{L P}\left(t^{\prime}\right)$, we have $\widehat{\mu}(Z)=\overleftarrow{\mu}_{t, t^{\prime}}^{*} Z$, see [34, Lemma 3.3.4] Correspondingly, denote $\widehat{\mu}$ by $\overleftarrow{\mu}_{t, t^{\prime}}^{*}$ for simplicity.

Consider the following set of Laurent polynomials

$$
\mathcal{L} \mathcal{P}\left(t ; t^{\prime}\right):=\mathcal{L} \mathcal{P}(t) \cap \overleftarrow{\mu}_{t^{\prime}, t}^{*} \mathcal{L P}\left(t^{\prime}\right)
$$

Then $\mathcal{L P}\left(t ; t^{\prime}\right)$ is a $\mathbb{k}$-algebra, such that $\overleftarrow{\mu}_{t, t^{\prime}}^{*} \mathcal{L P}\left(t ; t^{\prime}\right)=\mathcal{L} \mathcal{P}\left(t^{\prime} ; t\right)$

The following very useful result shows that certain mutation sequences swap pointedness and copointedness, where $t[-1]$ is a shifted seed constructed from a given seed $t$ (Definition 3.18).

Proposition 3.12 (Swap [34, Propositions 3.3.9 and 3.3.10]).

(1) For any $g, \eta \in M^{\circ}(t)$, we have $\eta \preceq_{t} g$ if and only if $\psi_{t[-1], t} \eta \succeq_{t} \psi_{t[-1], t} g$.

(2) Let there be given $Z \in \mathcal{L P}(t ; t[-1]) \subset \mathcal{L P}(t)$. Then $Z$ is $\eta$-copointed if and only if $\overleftarrow{\mu}_{t, t[-1]}^{*} Z$ is $\psi_{t[-1], t} \eta$-pointed.

Definition 3.13 (compatibility). If $Z$ belongs to $\mathcal{L P}\left(t ; t^{\prime}\right) \subset \mathcal{L P}(t)$, then $Z$ is said to be compatibly pointed at $t, t^{\prime}$ if it is $g$-pointed for some $g \in M^{\circ}(t)$, and $\overleftarrow{\mu}_{t, t^{\prime}}^{*} Z$ is $\phi_{t^{\prime}, t} g$-pointed.

If $Z$ belongs to $\mathcal{U}_{q}(t) \subset \mathcal{L P}(t)$, then $Z$ is said to be compatibly pointed at $\Delta^{+}$if it is compatibly pointed at $t, t^{\prime}$ for any $t^{\prime} \in \Delta^{+}$.

Let $S$ denote a set consisting of $g$-pointed functions $S_{g} \in \widehat{\mathcal{L P}}(t)$ for distinct $g \in M^{\circ}(t)$. If $S_{g}$ is compatibly pointed at $t, t^{\prime}$ for each $g$, we say $S$ is compatibly pointed at $t, t^{\prime}$, or the pointed sets $S$ and $\overleftarrow{\mu}_{t, t^{\prime}}^{*} S$ are (degree) compatible.

Thanks to [4, 14], we know that any given cluster monomial is compatibly pointed at all seeds. In particular, the degrees of its Laurent expansions at different seeds are related by tropical transformations. 
Definition 3.14 (codegree tropical transformation). For any seeds $t^{\prime}=\mu_{k} t, k \in I_{\text {uf }}$, we define the codegree tropical transformation $\phi_{t^{\prime}, t}^{\mathrm{op}}: M^{\circ}(t) \simeq M^{\circ}\left(t^{\prime}\right)$ as such that, for any $g=\left(g_{i}\right)_{i \in I} \in$ $M^{\circ}(t) \simeq \mathbb{Z}^{I}$, its image $\phi_{t^{\prime}, t}^{\mathrm{op}} g=\left(g_{i}^{\prime}\right)_{i \in I} \in M^{\circ}\left(t^{\prime}\right) \simeq \mathbb{Z}^{I}$ is given by

$$
g_{i}^{\prime}=\left\{\begin{array}{lll}
-g_{k}, & i=k, \\
g_{i}-b_{i k}\left[g_{k}\right]_{+}, & i \neq k, \quad b_{i k} \leq 0 \\
g_{i}-b_{i k}\left[-g_{k}\right]_{+}, & i \neq k, \quad b_{i k}>0 .
\end{array}\right.
$$

In general, we define the codegree tropical transformation $\phi_{t^{\prime}, t}^{\mathrm{op}}: M^{\circ}(t) \simeq M^{\circ}\left(t^{\prime}\right)$ as the composition of the codegree tropical transformations for adjacent seeds along the mutation sequence $\overleftarrow{\mu}$ from $t$ to $t^{\prime}$.

Let us justify our definition of the codegree tropical transformation.

To any given seed $t=\left(\widetilde{B}, \Lambda,\left(X_{i}\right)_{i \in I}\right)$, we associate the opposite seed defined to be $\iota(t):=$ $t^{\mathrm{op}}:=\left(-\widetilde{B},-\Lambda,\left(X_{i}\right)_{i \in I}\right)$. Then [34, Lemma 2.2.5] implies that, for any mutation sequence $\overleftarrow{\mu}$ we have $(\overleftarrow{\mu} t)^{\mathrm{op}}=\overleftarrow{\mu}\left(t^{\mathrm{op}}\right)$

Let us define $\iota: M^{\circ}(t) \simeq M^{\circ}\left(t^{\mathrm{op}}\right)$ as an isomorphism on $\mathbb{Z}^{I}$ such that $\iota\left(f_{i}(t)\right)=\iota\left(f_{i}\left(t^{\mathrm{op}}\right)\right)$. Correspondingly, by defining $\iota\left(X^{m}\right)=X^{m}$, we obtain natural $\mathbb{k}$-algebra anti-isomorphisms

$$
\begin{aligned}
& \iota: \quad \mathcal{L P}(t) \simeq \mathcal{L P}\left(t^{\mathrm{op}}\right), \\
& \iota: \widehat{\mathcal{L P}}(t) \simeq \widehat{\mathcal{L P}}\left(t^{\mathrm{op}}\right), \\
& \iota: \widehat{\mathcal{L P}}(t) \simeq \widehat{\mathcal{L P}}\left(t^{\mathrm{op}}\right) .
\end{aligned}
$$

Notice that $\iota: \mathcal{L P}(t) \simeq \mathcal{L P}\left(t^{\mathrm{op}}\right)$ induces an anti-isomorphism $\iota: \mathcal{F}(t) \simeq \mathcal{F}\left(t^{\mathrm{op}}\right)$.

For any given $k \in I_{\mathrm{uf}}$, we have $\mu_{k}\left(t^{\mathrm{op}}\right)=\left(\mu_{k} t\right)^{\mathrm{op}}$. It is straightforward to check the commutativity of the following diagram:

$$
\begin{array}{ccc}
\mathcal{F}(t) & \stackrel{\iota}{\rightarrow} & \mathcal{F}\left(t^{\mathrm{op}}\right) \\
\uparrow \mu_{k}^{*} & & \uparrow \mu_{k}^{*} \\
\mathcal{F}\left(\mu_{k} t\right) & \stackrel{\iota}{\rightarrow} & \mathcal{F}\left(\mu_{k}\left(t^{\mathrm{op}}\right)\right) .
\end{array}
$$

In particular, $\iota\left(\mu_{k}^{*} X_{i}\left(\mu_{k} t\right)\right)=\mu_{k}^{*}\left(\iota X_{i}\left(\mu_{k} t\right)\right)$ is given by $X_{i}\left(t^{\text {op }}\right)$ if $i \neq k$, or

$$
X\left(t^{\mathrm{op}}\right)^{-f_{k}\left(t^{\mathrm{op}}\right)+\sum_{j}\left[-b_{j k}\right]_{+} f_{j}\left(t^{\mathrm{op}}\right)}+X\left(t^{\mathrm{op}}\right)^{-f_{k}\left(t^{\mathrm{op}}\right)+\sum_{i}\left[b_{i k}\right]_{+} f_{i}\left(t^{\mathrm{op}}\right)}
$$

if $i=k$.

Notice that $Y(t)^{n}=X^{\widetilde{B} n}$ while $Y\left(t^{\text {op }}\right)^{n}=X^{-\widetilde{B} n}$. It follows that $Z \in \widehat{\mathcal{L P}}(t)$ is $g$-pointed if and only if $\iota Z \in \widehat{\mathcal{L P}}\left(t^{\mathrm{op}}\right)$ is $g$-copointed. We have the following result.

Lemma 3.15. Let there be given seeds $t^{\prime}=\overleftarrow{\mu}_{t^{\prime}, t} t$. Then the codegree tropical transformation $\phi_{t^{\prime}, t}^{\mathrm{op}}: M^{\circ}(t) \simeq M^{\circ}\left(t^{\prime}\right)$ equals the composition $M^{\circ}(t) \stackrel{\iota}{\rightarrow} M^{\circ}\left(t^{\mathrm{op}}\right) \stackrel{\phi_{\left(t^{\prime}\right) \mathrm{op}, t^{\mathrm{op}}}}{\rightarrow} M^{\circ}\left(\left(t^{\prime}\right)^{\mathrm{op}}\right) \stackrel{\iota}{\rightarrow} M^{\circ}\left(t^{\prime}\right)$. In particular, it is independent of the choice of $\overleftarrow{\mu}_{t^{\prime}, t}$.

Proof. By the commutativity between $\iota$ and mutations, it suffices to check the claim for adjacent seeds $t^{\prime}=\mu_{k} t$, which follows from definition.

Notice that we have $\mathcal{L P}\left(t ; t^{\prime}\right)=\iota \mathcal{L} \mathcal{P}\left(t^{\mathrm{op}} ;\left(t^{\prime}\right)^{\mathrm{op}}\right)$ and $\mathcal{U}_{q}(t)=\iota\left(\mathcal{U}_{q}\left(t^{\mathrm{op}}\right)\right)$ by the commutativity between $\iota$ and mutations.

Definition 3.16 (codegree compatibility). If $Z$ belongs to $\mathcal{L P}\left(t ; t^{\prime}\right) \subset \mathcal{L P}(t)$, then $Z$ is said to be compatibly copointed at $t, t^{\prime}$ if it is $\eta$-copointed for some $\eta \in M^{\circ}(t)$, and $\overleftarrow{\mu}_{t, t^{\prime}}^{*} Z$ is $\phi_{t^{\prime}, t}^{\mathrm{op}} \eta$-copointed. 
If $Z$ belongs to $\mathcal{U}_{q}(t) \subset \mathcal{L P}(t)$, then $Z$ is said to be compatibly copointed at $\Delta^{+}$if it is compatibly copointed at $t, t^{\prime}$ for any $t^{\prime} \in \Delta^{+}$.

Let $S$ denote a set consisting of $\eta$-copointed elements $S^{\eta} \in \widetilde{\mathcal{L P}}(t)$ for distinct $\eta \in M^{\circ}(t)$. If $S^{\eta}$ are compatibly copointed at $t, t^{\prime}$ for all $\eta$, we say $S$ is compatibly copointed at $t, t^{\prime}$, or the copointed sets $S$ and $\overleftarrow{\mu}_{t, t^{\prime}}^{*} S$ are (codegree) compatible.

Remark 3.17. We refer the reader to [20, Section 3.5] for a categorical view. In their setting, to study cluster algebras arising from quantum unipotent cells, one considers certain module category of the corresponding quiver Hecke algebras and localize it at the simple objects corresponding to the frozen variables. Then the degrees and codegrees, together with the tropical transformations, can be calculated by taking dual objects in the localized module category.

\subsection{Injective-reachability and distinguished functions}

Let $\sigma$ denote a permutation of $I_{\mathrm{uf}}$. For any mutation sequence $\overleftarrow{\mu}=\mu_{k_{r}} \cdots \mu_{k_{1}}$, we define $\sigma \overleftarrow{\mu}=\mu_{\sigma k_{r}} \cdots \mu_{\sigma k_{1}}$

Let $\operatorname{pr}_{I_{\mathrm{uf}}}$ and $\mathrm{pr}_{I_{\mathrm{f}}}$ denote the natural projection from $\mathbb{Z}^{I}$ to $\mathbb{Z}^{I_{\mathrm{uf}}}$ and $\mathbb{Z}^{I_{\mathrm{f}}}$ respectively.

Definition 3.18 ([33, Definition 5.1.1]). A seed $t$ is said to be injective-reachable if there exists a mutation sequence $\overleftarrow{\mu}=\overleftarrow{\mu}_{t^{\prime}, t}$ and a permutation $\sigma$ of $I_{\mathrm{uf}}$, such that the seed $t^{\prime}=\overleftarrow{\mu}_{t^{\prime}, t} t$ satisfies $b_{\sigma i, \sigma j}\left(t^{\prime}\right)=b_{i j}(t)$ for $i, j \in I_{\mathrm{uf}}$ and, for any $k \in I_{\mathrm{uf}}$,

$$
\operatorname{deg}^{t} \overleftarrow{\mu}_{t^{\prime}, t}^{*} X_{\sigma k}\left(t^{\prime}\right)=-f_{k}+u_{k}
$$

for some $u_{k} \in \mathbb{Z}^{I_{\mathrm{f}}}$.

In this case, we denote $t^{\prime}=t[1]$ and say it is shifted from $t$ (by [1]) with the permutation $\sigma$. Similarly, we denote $t=t^{\prime}[-1]$ and say it is the shifted from $t^{\prime}$ (by [-1]) with the permutation $\sigma^{-1}$.

We recall that, up to a permutation of vertices, a seed is determined by the degrees (extended $g$-vectors) of its cluster variables, see [14] for an interpretation in terms of chambers. In particular, the shifted seed $t[1]$ is unique up to a permutation.

Let there be given an injective-reachable seed $t$. Recursively, we construct a chain of seeds $\{t[d] \mid d \in \mathbb{Z}\}$ called an injective-reachable chain, such that $t[d]=\left(\sigma^{d-1} \overleftarrow{\mu}\right) t[d-1]$, see $[33$ Definition 5.2.1]. In particular, we have $t=\left(\sigma^{-1} \overleftarrow{\mu}\right) t[-1]$

We denote $I_{k}(t)=\overleftarrow{\mu}_{t[1], t}^{*} X_{\sigma k}(t[1])$ and $P_{k}(t)=\overleftarrow{\mu}_{t[-1], t}^{*} X_{\sigma^{-1}(k)}(t[-1])$. For any $d \in \mathbb{N}^{I_{\text {uf }}}$ define the cluster monomial $I(t)^{d}:=\left[\prod_{k} I_{k}(t)^{d_{k}}\right]^{t}$ and $P(t)^{d}:=\left[\prod_{k} P_{k}(t)^{d_{k}}\right]^{t}$.

Since a quantum cluster monomial is pointed, it is also copointed by [8] (we can also see this using the map $\iota$ ). It follows that $I(t)^{d}=\left\{\prod_{k} I_{k}(t)^{d_{k}}\right\}^{t}$ and $P(t)^{d}=\left\{\prod_{k} P_{k}(t)^{d_{k}}\right\}^{t}$.

Notice that if $t$ is injective-reachable, then so is any seed $t^{\prime} \in \Delta^{+}$. Such property is equivalent to the existence of a green to red sequence. See [33, 34] for more details.

For any $g=\left(g_{i}\right)_{i \in I} \in \mathbb{Z}^{I} \simeq M^{\circ}(t)$, denote $[g]_{+}=\left(\left[g_{i}\right]_{+}\right)_{i \in I}$. We have the following $g$-pointed element in $\mathcal{L P}(t)$ :

$$
\mathbf{I}_{g}^{t}=\left[p_{g} * X(t)^{[g]_{+}} * I(t)^{\left[-\operatorname{pr}_{I_{\mathrm{uf}}} g\right]_{+}}\right]^{t}
$$

for some frozen factor $p_{g} \in \mathcal{P}$. Define the following set of distinguished pointed functions

$$
\mathbf{I}^{t}:=\left\{\mathbf{I}_{g}^{t} \mid g \in M^{\circ}(t)\right\} .
$$

Denote $t^{\prime}=t[1]$. By (3.4), the linear map $\psi_{t, t^{\prime}}: M^{\circ}\left(t^{\prime}\right) \simeq M^{\circ}(t)$ is determined by

$$
\begin{aligned}
& \psi_{t, t^{\prime}}\left(f_{\sigma k}^{\prime}\right)=-f_{k}+u_{k}, \quad u_{k} \in \mathbb{Z}^{I_{\mathrm{f}}}, \quad k \in I_{\mathrm{uf}}, \\
& \psi_{t, t^{\prime}}\left(f_{i}^{\prime}\right)=f_{i}, \quad i \in I_{\mathrm{f}} .
\end{aligned}
$$


Using Proposition 3.12, we deduce that

$$
\operatorname{codeg}^{t[1]} P_{\sigma k}(t[1])=\operatorname{codeg}^{t[1]} \overleftarrow{\mu}_{t, t[1]}^{*} X_{k}(t)=\psi_{t, t[1]}^{-1} f_{k}=-f_{\sigma k}(t[1])+u_{k}
$$

Notice that (3.5) appears in [33, equation (18)] as an assumption. Replacing $t$ by $t[-1]$ in the above argument, we obtain

$$
\operatorname{codeg}^{t} P_{\sigma k}(t)=-f_{\sigma k}+u_{k}^{\prime}
$$

for any $k \in I_{\mathrm{uf}}$ and some $u_{k}^{\prime} \in \mathbb{Z}^{I_{\mathrm{f}}}$.

Correspondingly, for any $\eta \in \mathbb{Z}^{I} \simeq M^{\circ}(t)$, we have the following $\eta$-copointed element in $\mathcal{L P}(t)$ :

$$
\mathbf{P}^{t, \eta}=\left\{P(t)^{\left[-\operatorname{pr}_{I_{\mathrm{uf}}} \eta\right]+} * X(t)^{[\eta]+} * p^{\eta}\right\}^{t}
$$

for some frozen factor $p^{\eta} \in \mathcal{P}$. Define the following set of distinguished copointed functions

$$
\mathbf{P}^{t}:=\left\{\mathbf{P}^{t, \eta} \mid \eta \in M^{\circ}(t)\right\} .
$$

The two kinds of distinguished functions are related by the following result. At a categorical level, it can be viewed as the duality between injective representations and projective representations for a pair of opposite quivers, see [33, Section 5.3] for more discussion.

Lemma 3.19. Denote $\overleftarrow{\mu}=\overleftarrow{\mu}_{t[1], t}$. The following claims are true.

(1) For any $k \in I_{\mathrm{uf}}$, we have $\iota P_{k}(t)=I_{k}\left(t^{\mathrm{op}}\right)$.

(2) We have $t[-1]^{\mathrm{op}}=\left(t^{\mathrm{op}}\right)[1]=\left(\sigma^{-1} \overleftarrow{\mu}\right)^{-1} t^{\mathrm{op}}$, which is shifted from $t^{\mathrm{op}}$ with the permutation $\sigma^{-1}$.

(3) We have $t[1]^{\mathrm{op}}=\left(t^{\mathrm{op}}\right)[-1]=\overleftarrow{\mu} t^{\mathrm{op}}$, which is shifted from $t^{\mathrm{op}}$ with the permutation $\sigma$

Proof. (1) Recall that $\iota P_{k}(t)$ is a quantum cluster variable contained in $\mathcal{L P}\left(t^{\text {op }}\right)$. By (3.6), $\iota P_{k}(t)$ is pointed at $-f_{k}+u$ for some $u \in \mathbb{Z}^{I_{\mathrm{f}}}$. The claim follows.

(2) Denote $\overleftarrow{\nu}=\left(\sigma^{-1} \overleftarrow{\mu}\right)^{-1}$ for simplicity. By the commutativity between mutations and $\iota$ we have $t[-1]^{\mathrm{op}}=(\overleftarrow{\nu} t)^{\mathrm{op}}=\overleftarrow{\nu} t^{\mathrm{op}}$

The seed $t[-1]^{\mathrm{op}}$ has the principal $B$-matrix given by $b_{i j}\left(t[-1]^{\mathrm{op}}\right)=-b_{i j}(t[-1])=-b_{\sigma i, \sigma j}$, $i, j \in I_{\text {uf }}$. Using the commutativity relation (3.3) between $\iota: \mathcal{F}(t) \simeq \mathcal{F}\left(t^{\mathrm{op}}\right), \iota: \mathcal{F}(\overleftarrow{\nu} t) \simeq$ $\mathcal{F}\left((\overleftarrow{\nu} t)^{\text {op }}\right)$ and mutations, its cluster variables have the following Laurent expansion in $\mathcal{L} \mathcal{P}\left(t^{\text {op }}\right)$ :

$$
(\overleftarrow{\nu})^{*}\left(X_{\sigma^{-1} k}\left(\overleftarrow{\nu} t^{\mathrm{op}}\right)\right)=\iota\left((\overleftarrow{\nu})^{*} \iota^{-1} X_{\sigma^{-1} k}\left(\overleftarrow{\nu} t^{\mathrm{op}}\right)\right)=\iota\left((\overleftarrow{\nu})^{*} X_{\sigma^{-1} k}(\overleftarrow{\nu} t)\right)=\iota\left(P_{k}(t)\right)
$$

$k \in I_{\text {uf }}$, which are pointed at $-f_{k}+u, u \in \mathbb{Z}^{I_{\mathrm{f}}}$. It follows that $t[-1]^{\text {op }}$ is a shifted seed $t^{\text {op }}[1]$ with the permutation $\sigma^{-1}$.

(3) Notice that $t[1]^{\mathrm{op}}=(\overleftarrow{\mu} t)^{\mathrm{op}}=\overleftarrow{\mu}\left(t^{\mathrm{op}}\right)$ by the commutativity between mutations and $\iota$ Since $\overleftarrow{\mu}^{-1} t^{\mathrm{op}}=t^{\mathrm{op}}[1]$ with the permutation $\sigma^{-1}$ by $(2)$, we have $\overleftarrow{\mu} t^{\mathrm{op}}=t^{\mathrm{op}}[-1]$ with the permutation $\sigma$.

Example 3.20. Let us continue Example 3.8. Ignore the Lambda matrices for simplicity and set $v=1$. Then the cluster algebra have five cluster variables in $\mathcal{L P}\left(t_{0}\right)$ :

$$
X_{1}, \quad X_{2}, \quad P_{2}=\frac{X_{1}+1}{X_{2}}, \quad P_{1}=I_{2}=\frac{X_{1}+1+X_{2}}{X_{1} X_{2}}, \quad I_{1}=\frac{1+X_{2}}{X_{1}} .
$$

Take the seed $t_{0}=\left(B,\left(X_{1}, X_{2}\right)\right)$. Choose the mutation sequence $\overleftarrow{\mu}=\mu_{2} \mu_{1} \mu_{2}$ (read from right to left). Then

$$
t_{0}[1]:=\overleftarrow{\mu} t_{0}=\left(-B,\left(I_{2}, I_{1}\right)\right)
$$


is shifted from $t_{0}$ by [1] with the unique non-trivial permutation $\sigma$ of $I=\{1,2\}$. Notice that $\left(\sigma^{-1} \overleftarrow{\mu}\right)^{-1}=\mu_{1} \mu_{2} \mu_{1}$. Similarly

$$
t_{0}[-1]:=\left(\sigma^{-1} \overleftarrow{\mu}\right)^{-1} t_{0}=\left(-B,\left(P_{2}, P_{1}\right)\right)
$$

is shifted from $t_{0}$ by $[-1]$ with $\sigma^{-1}$.

It follows that

$$
\begin{aligned}
& t_{0}^{\mathrm{op}}=\left(-B,\left(\iota X_{1}, \iota X_{2}\right)\right)=\left(-B,\left(X_{1}\left(t_{0}^{\mathrm{op}}\right), X_{2}\left(t_{0}^{\mathrm{op}}\right)\right)\right) \\
& t_{0}[1]^{\mathrm{op}}=\left(B,\left(\iota I_{2}, \iota I_{1}\right)\right)=\left(B,\left(P_{2}\left(t_{0}^{\mathrm{op}}\right), P_{1}\left(t_{0}^{\mathrm{op}}\right)\right)\right)=\overleftarrow{\mu} t_{0}^{\mathrm{op}} \\
& t_{0}[-1]^{\mathrm{op}}=\left(B,\left(\iota P_{2}, \iota P_{1}\right)\right)=\left(B,\left(I_{2}\left(t_{0}^{\mathrm{op}}\right), I_{1}\left(t_{0}^{\mathrm{op}}\right)\right)\right)=\left(\sigma^{-1} \overleftarrow{\mu}\right)^{-1} t_{0}^{\mathrm{op}}
\end{aligned}
$$

We see that $t_{0}[-1]^{\text {op }}$ is shifted from $t_{0}^{\text {op }}$ by [1] with the permutation $\sigma^{-1}$, which we denote by $t_{0}[-1]^{\mathrm{op}}=t_{0}^{\mathrm{op}}[1]$, and $t_{0}[1]^{\mathrm{op}}$ is shifted from $t_{0}^{\mathrm{op}}$ by $[-1]$ with the permutation $\sigma$, which we denote by $t_{0}[1]^{\mathrm{op}}=t_{0}^{\mathrm{op}}[-1]$.

Lemma 3.21 (substitution).

(1) Assume that $\left[X(t)^{d} * I(t)^{d^{\prime}}\right]^{t}$ is $\left(\prec_{t}, \mathbf{m}\right)$-unitriangular to $\mathbf{I}^{t}$ for any $d \in \mathbb{N}^{I_{\mathrm{uf}}} \oplus \mathbb{Z}^{I_{\mathrm{f}}}$ and $d^{\prime} \in \mathbb{N}^{I_{\mathrm{uf}}}$. If $Z$ is $\left(\prec_{t}, \mathbf{m}\right)$-unitriangular to $\mathbf{I}^{t}$, then the normalized products $\left[X(t)^{d} * Z *\right.$ $\left.I(t)^{d^{\prime}}\right]^{t}$ are $\left(\prec_{t}, \mathbf{m}\right)$-unitriangular to $\mathbf{I}^{t}$ too [33, Lemma 6.2.4].

(2) Assume that $\left\{P(t)^{d^{\prime}} * X(t)^{d}\right\}^{t}$ is codegree $\left(\succ_{t}, \mathbf{m}\right)$-unitriangular to $\mathbf{P}^{t}$ for any $d \in \mathbb{N}^{I_{\mathrm{uf}}} \oplus \mathbb{Z}^{I_{\mathrm{f}}}$ and $d^{\prime} \in \mathbb{N}^{I_{\mathrm{uf}}}$. If $Z$ is codegree $\left(\succ_{t}, \mathbf{m}\right)$-unitriangular to $\mathbf{P}^{t}$, then the codegree normalized products $\left\{P(t)^{d^{\prime}} * Z * X(t)^{d}\right\}^{t}$ are codegree $\left(\succ_{t}, \mathbf{m}\right)$-unitriangular to $\mathbf{P}^{t}$ too.

Proof. (1) has been proved in [33]. We can prove (2) using similar arguments as those for (1), or deduce (2) from (1) by using the map $\iota$.

We have the following relation between degree and codegree tropical transformations.

Proposition 3.22. For any $t, t^{\prime} \in \Delta^{+}$, the following diagram commutes:

$$
\begin{array}{ccc}
M^{\circ}(t[1]) & \stackrel{\psi_{t, t[1]}}{\longrightarrow} & M^{\circ}(t) \\
\downarrow \phi_{t^{\prime}[1], t[1]}^{\mathrm{op}} & & \downarrow \phi_{t^{\prime}, t} \\
M^{\circ}\left(t^{\prime}[1]\right) & \stackrel{\psi_{t^{\prime}, t^{\prime}[1]}}{\longrightarrow} & M^{\circ}\left(t^{\prime}\right) .
\end{array}
$$

Proof. It suffices to check the claim for the case $t^{\prime}=\mu_{k} t, k \in I_{\mathrm{uf}}$. Notice that, in this case, we have $t^{\prime}[1]=\mu_{\sigma k}(t[1])$ and $\overleftarrow{\mu}_{t, t^{\prime}}^{*} I_{i}(t)=I_{i}\left(t^{\prime}\right)$ for $i \neq k$, see [33, Proposition 5.1.4].

Notice that, all $u \in \mathbb{Z}^{I_{\mathrm{f}}}$ are invariant under the maps in the diagrams. In view of the piecewise linearity of $\phi_{t^{\prime}, t}$ and $\phi_{t^{\prime}[1], t[1]}^{\mathrm{op}}$, it remains to check the claim that, for $i \in I_{\mathrm{uf}}$,

$$
\phi_{t^{\prime}, t} \psi_{t, t[1]}\left( \pm f_{\sigma i}(t[1])\right)=\psi_{t^{\prime}, t^{\prime}[1]} \phi_{t^{\prime}[1], t[1]}^{\mathrm{op}}\left( \pm f_{\sigma i}(t[1])\right) .
$$

(i) By definition, for $i \neq k$ in $I_{\text {uf }}$, we have

$$
\phi_{t^{\prime}, t} \psi_{t, t[1]}\left(f_{\sigma i}(t[1])\right)=\operatorname{deg}^{t^{\prime}} \overleftarrow{\mu}_{t, t^{\prime}}^{*} I_{i}(t)=\operatorname{deg}^{t^{\prime}} I_{i}\left(t^{\prime}\right)
$$

and also

$$
\psi_{t^{\prime}, t^{\prime}[1]} \phi_{t^{\prime}[1], t[1]}^{\mathrm{op}}\left(f_{\sigma i}(t[1])\right)=\psi_{t^{\prime}, t^{\prime}[1]}\left(f_{\sigma i}\left(t^{\prime}[1]\right)\right)=\operatorname{deg}^{t^{\prime}} I_{i}\left(t^{\prime}\right) .
$$

It follows that these two vectors in $M^{\circ}\left(t^{\prime}\right)$ agree. 
(ii) For the non-trivial case $i=k$, we have

$$
\phi_{t^{\prime}, t} \psi_{t, t[1]}\left(f_{\sigma k}(t[1])\right)=\operatorname{deg}^{t^{\prime}} \overleftarrow{\mu}_{t, t^{\prime}}^{*} I_{k}(t)
$$

Notice that $I_{k}(t)$ and $I_{k}\left(t^{\prime}\right)$ are related by an exchange relation for the seeds $\left(t[1], t^{\prime}[1]\right)$. It follows that we have

$$
\operatorname{deg}^{t^{\prime}} \overleftarrow{\mu}_{t, t^{\prime}}^{*} I_{k}(t)=-\operatorname{deg}^{t^{\prime}} I_{k}\left(t^{\prime}\right)+\sum_{i \in I_{\mathrm{uf}}}\left[b_{i k}\left(t^{\prime}\right)\right]_{+} \operatorname{deg}^{t^{\prime}} I_{i}\left(t^{\prime}\right)+\sum_{s \in I_{\mathrm{f}}}\left[b_{s, \sigma k}\left(t^{\prime}[1]\right)\right]_{+} f_{s}
$$

see [33, equation (14)].

On the other hand, we have the following computation

$$
\begin{aligned}
\psi_{t^{\prime}, t^{\prime}[1]} & \phi_{t^{\prime}[1], t[1]}^{\mathrm{op}}\left(f_{\sigma k}(t[1])\right) \\
& =\psi_{t^{\prime}, t^{\prime}[1]}\left(-f_{\sigma k}\left(t^{\prime}[1]\right)+\sum_{i \in I_{\mathrm{uf}}}\left[-b_{\sigma i, \sigma k}(t[1])\right]_{+} f_{\sigma i}\left(t^{\prime}[1]\right)+\sum_{s \in I_{\mathrm{f}}}\left[-b_{s, \sigma k}(t[1])\right]_{+} f_{s}\right) \\
& =-\operatorname{deg}^{t^{\prime}} I_{k}\left(t^{\prime}\right)+\sum_{i \in I_{\mathrm{uf}}}\left[-b_{\sigma i, \sigma k}(t[1])\right]_{+} \operatorname{deg}^{t^{\prime}} I_{i}\left(t^{\prime}\right)+\sum_{s \in I_{\mathrm{f}}}\left[-b_{s, \sigma k}(t[1])\right]_{+} f_{s} \\
& =-\operatorname{deg}^{t^{\prime}} I_{k}\left(t^{\prime}\right)+\sum_{i \in I_{\mathrm{uf}}}\left[b_{i k}\left(t^{\prime}\right)\right]_{+} \operatorname{deg}^{t^{\prime}} I_{i}\left(t^{\prime}\right)+\sum_{s \in I_{\mathrm{f}}}\left[b_{s, \sigma k}\left(t^{\prime}[1]\right)\right]_{+} f_{s} .
\end{aligned}
$$

The desired equality follows:

$$
\phi_{t^{\prime}, t} \psi_{t, t[1]}\left(f_{\sigma k}(t[1])\right)=\psi_{t^{\prime}, t^{\prime}[1]} \phi_{t^{\prime}[1], t[1]}^{\mathrm{op}}\left(f_{\sigma k}(t[1])\right) .
$$

(iii) By (3.4) and the linearity of $\psi_{t, t[1]}, \psi_{t^{\prime}, t^{\prime}[1]}$, for $i \neq k$ in $I_{\mathrm{uf}}$, we have

$$
\phi_{t^{\prime}, t} \psi_{t, t[1]}\left(-f_{\sigma i}(t[1])\right)=\phi_{t^{\prime}, t}\left(f_{i}(t)-u_{i}\right)=f_{i}\left(t^{\prime}\right)-u_{i}
$$

and also

$$
\psi_{t^{\prime}, t^{\prime}[1]} \phi_{t^{\prime}[1], t[1]}^{\mathrm{op}}\left(-f_{\sigma i}(t[1])\right)=\psi_{t^{\prime}, t^{\prime}[1]}\left(-f_{\sigma i}\left(t^{\prime}[1]\right)\right)=-\operatorname{deg}^{t^{\prime}} I_{i}\left(t^{\prime}\right) .
$$

Then (3.4) implies that the two vectors in $M^{\circ}\left(t^{\prime}\right)$ agree.

(iv) For the non-trivial case $i=k$, the linearity of $\psi_{t, t[1]}$ implies

$$
\psi_{t, t[1]}\left(-f_{\sigma k}(t[1])\right)=-\operatorname{deg}^{t} I_{k}(t) .
$$

Notice that $I_{k}(t)$ and $I_{k}\left(t^{\prime}\right)$ are related by an exchange relation for the seeds $\left(t[1], t^{\prime}[1]\right)$. It follows that we have

$$
\operatorname{deg}^{t} \overleftarrow{\mu}_{t^{\prime}, t}^{*} I_{k}\left(t^{\prime}\right)=-\operatorname{deg}^{t} I_{k}(t)+\sum_{i \in I_{\mathrm{uf}}}\left[b_{i k}(t)\right]_{+} \operatorname{deg}^{t} I_{i}(t)+\sum_{s \in I_{\mathrm{f}}}\left[b_{s, \sigma k}(t[1])\right]_{+} f_{s}
$$

see [33, equation (14)]. Consequently, we get

$$
\psi_{t, t[1]}\left(-f_{\sigma k}(t[1])\right)=\operatorname{deg}^{t} \overleftarrow{\mu}_{t^{\prime}, t}^{*} I_{k}\left(t^{\prime}\right)-\sum_{i \in I_{\mathrm{uf}}}\left[b_{i k}(t)\right]_{+} \operatorname{deg}^{t} I_{i}(t)-\sum_{s \in I_{\mathrm{f}}}\left[b_{s, \sigma k}(t[1])\right]_{+} f_{s}
$$

On the other hand, we compute that

$$
\psi_{t^{\prime}, t^{\prime}[1]} \phi_{t^{\prime}[1], t[1]}^{\mathrm{op}}\left(-f_{\sigma k}(t[1])\right)
$$




$$
\begin{aligned}
& =\psi_{t^{\prime}, t^{\prime}[1]}\left(f_{\sigma k}\left(t^{\prime}[1]\right)-\sum_{i \in I_{\mathrm{uf}}}\left[b_{\sigma i, \sigma k}(t[1])\right]_{+} f_{\sigma i}\left(t^{\prime}[1]\right)-\sum_{s \in I_{\mathrm{f}}}\left[b_{s, \sigma k}(t[1])\right]_{+} f_{s}\right) \\
& =\operatorname{deg}^{t^{\prime}} I_{k}\left(t^{\prime}\right)-\sum_{i \in I_{\mathrm{uf}}}\left[b_{\sigma i, \sigma k}(t[1])\right]_{+} \operatorname{deg}^{t^{\prime}} I_{i}\left(t^{\prime}\right)-\sum_{s \in I_{\mathrm{f}}}\left[b_{s, \sigma k}(t[1])\right]_{+} f_{s} \\
& =\operatorname{deg}^{t^{\prime}} I_{k}\left(t^{\prime}\right)-\sum_{i \in I_{\mathrm{uf}}}\left[b_{i k}(t)\right]_{+} \operatorname{deg}^{t^{\prime}} I_{i}\left(t^{\prime}\right)-\sum_{s \in I_{\mathrm{f}}}\left[b_{s, \sigma k}(t[1])\right]_{+} f_{s} .
\end{aligned}
$$

Further applying $\phi_{t, t^{\prime}}$ to both sides, we obtain

$$
\begin{aligned}
& \phi_{t, t^{\prime}} \psi_{t^{\prime}, t^{\prime}[1]} \phi_{t^{\prime}[1], t[1]}^{\mathrm{op}}\left(-f_{\sigma k}(t[1])\right) \\
& \quad=\operatorname{deg}^{t} \overleftarrow{\mu}_{t^{\prime}, t}^{*} I_{k}\left(t^{\prime}\right)-\sum_{i \in I_{\mathrm{uf}}}\left[b_{i k}(t)\right]_{+} \operatorname{deg}^{t} I_{i}(t)-\sum_{s \in I_{\mathrm{f}}}\left[b_{s, \sigma k}(t[1])\right]_{+} f_{s}
\end{aligned}
$$

Consequently, we have

$$
\psi_{t, t[1]}\left(-f_{\sigma k}(t[1])\right)=\phi_{t, t^{\prime}} \psi_{t^{\prime}, t^{\prime}[1]} \phi_{t^{\prime}[1], t[1]}^{\mathrm{op}}\left(-f_{\sigma k}(t[1])\right)
$$

We obtain the claim observing that $\phi_{t, t^{\prime}}=\phi_{t^{\prime}, t}^{-1}$.

Consequently, we obtain a relation between the degree compatibility and the codegree compatibility, which will be useful for studying properties of double triangular bases (Proposition 4.9).

Proposition 3.23. Let there be given seeds $t, t^{\prime} \in \Delta^{+}$and $Z \in \mathcal{L P}(t) \cap \overleftarrow{\mu}_{t^{\prime}, t}^{*} \mathcal{L P}\left(t^{\prime}\right) \cap \overleftarrow{\mu}_{t[1], t}^{*} \mathcal{L P}(t[1])$ $\cap \overleftarrow{\mu}_{t^{\prime}[1], t}^{*} \mathcal{L P}\left(t^{\prime}[1]\right)$. Then $Z$ is compatibly copointed at $t[1], t^{\prime}[1]$ if and only if it is compatibly pointed at $t, t^{\prime}$.

Proof. By Proposition 3.12, $\overleftarrow{\mu}_{t, t[1]}^{*} Z$ is $\eta$-copointed in $\mathcal{L P}(t[1])$ if and only if $Z$ is $\psi_{t, t[1]} \eta$-pointed in $\mathcal{L P}(t)$, and similar statements hold in $\mathcal{L P}\left(t^{\prime}[1]\right)$ and $\mathcal{L P}\left(t^{\prime}\right)$. Let us explain how the claim follows from Proposition 3.22.

First, assume that $Z$ is compatibly copointed at $t[1], t^{\prime}[1]$. This means that there exists some $\eta$ such that $\overleftarrow{\mu}_{t, t[1]}^{*} Z$ is $\eta$-copointed in $\mathcal{L} \mathcal{P}(t[1])$ and $\overleftarrow{\mu}_{t, t^{\prime}[1]}^{*} Z$ is $\phi_{t^{\prime}[1], t[1]}^{\mathrm{op}} \eta$-copointed in $\mathcal{L P}\left(t^{\prime}[1]\right)$. Then Proposition 3.12 implies that $Z$ is $\psi_{t, t[1]} \eta$-pointed in $\mathcal{L P}(t)$ and $\overleftarrow{\mu}_{t, t^{\prime}}^{*} Z=$ $\overleftarrow{\mu}_{t^{\prime}[1], t^{\prime}}^{*}\left(\overleftarrow{\mu}_{t, t^{\prime}[1]}^{*} Z\right)$ is $\psi_{t^{\prime}, t^{\prime}[1]}\left(\phi_{t^{\prime}[1], t[1]}^{\text {op }} \eta\right)$-pointed in $\mathcal{L P}\left(t^{\prime}\right)$. In addition, Proposition 3.22 implies that $\psi_{t^{\prime}, t^{\prime}[1]}\left(\phi_{t^{\prime}[1], t[1]}^{\mathrm{op}} \eta\right)=\phi_{t^{\prime}, t}\left(\psi_{t, t[1]} \eta\right)$. Therefore, $Z$ is compatibly pointed at $t, t^{\prime}$.

Conversely, assume that $Z$ is compatibly pointed at $t, t^{\prime}$. This means that there exists some $g$ such that $Z$ is $g$-pointed in $\mathcal{L P}(t)$ and $\overleftarrow{\mu}_{t, t^{\prime}}^{*} Z$ is $\phi_{t^{\prime}, t} g$-pointed in $\mathcal{L P}\left(t^{\prime}\right)$. Then Proposition 3.12 implies that $\overleftarrow{\mu}_{t, t[1]}^{*} Z$ is $\psi_{t, t[1]}^{-1} g$-copointed in $\mathcal{L P}(t[1])$ and $\overleftarrow{\mu}_{t, t^{\prime}[1]}^{*} Z$ is $\psi_{t^{\prime}, t^{\prime}[1]}^{-1}\left(\phi_{t^{\prime}, t} g\right)$-copointed in $\mathcal{L} \mathcal{P}\left(t^{\prime}[1]\right)$. In addition, Proposition 3.22 implies that $\phi_{t^{\prime}[1], t[1]}^{\mathrm{op}}\left(\psi_{t, t[1]}^{-1} g\right)=\psi_{t^{\prime}, t^{\prime}[1]}^{-1}\left(\phi_{t^{\prime}, t} g\right)$. Therefore, $Z$ is compatibly copointed at $t[1], t^{\prime}[1]$.

\section{Bidegrees and bases}

Let there be given an injective-reachable quantum seed $t$ and a subalgebra $\mathrm{A}(t) \subset \mathcal{U}_{q}(t)$. Assume that $\mathrm{A}(t)$ possesses a $\mathbb{k}$-basis $\mathbf{L}$. Then $\mathrm{A}(t)$ naturally gives rise to a subalgebra $\mathrm{A}\left(t^{\prime}\right):=$ $\overleftarrow{\mu}_{t, t^{\prime}}^{*} \mathrm{~A}(t) \subset \mathcal{U}_{q}\left(t^{\prime}\right)=\overleftarrow{\mu}_{t, t^{\prime}}^{*} \mathcal{U}_{q}(t)$, and $\mathbf{L}$ naturally gives rise to a basis $\overleftarrow{\mu}_{t, t^{\prime}}^{*} \mathbf{L}$ of $\mathrm{A}\left(t^{\prime}\right)$. We sometimes omit the symbols $t, t^{\prime}$, identifying $\mathrm{A}(t)$ and $\mathrm{A}\left(t^{\prime}\right), \mathbf{L}$ and $\overleftarrow{\mu}_{t, t^{\prime}}^{*} \mathbf{L}$ 


\subsection{Bases with different properties}

Definition 4.1 (degree-triangular basis). A $\mathbb{k}$-basis $\mathbf{L}$ of $\mathrm{A}(t)$ is said to be a degree-triangular basis with respect to $t$ if the following conditions hold:

(1) $X_{i}(t) \in \mathbf{L}$ for $i \in I$.

(2) Bar-invariance: $\mathbf{L}$ is invariant under the bar involution.

(3) Degree parametrization: $\mathbf{L}$ is $M^{\circ}(t)$-pointed, i.e., it takes the form $\mathbf{L}=\left\{\mathbf{L}_{g} \mid g \in M^{\circ}(t)\right\}$ such that $\mathbf{L}_{g}$ is $g$-pointed.

(4) Degree triangularity: For any basis element $\mathbf{L}_{g}, i \in I$, the decomposition of the pointed function $\left[X_{i}(t) * \mathbf{L}_{g}\right]^{t}$ in terms of $\mathbf{L}$ is degree $\left(\prec_{t}, \mathbf{m}\right)$-unitriangular:

$$
\left[X_{i}(t) * \mathbf{L}_{g}\right]^{t}=\sum_{g^{\prime} \varliminf_{t} g+f_{i}} b_{g^{\prime}} \mathbf{L}_{g^{\prime}}
$$

where $b_{g+f_{i}}=1, b_{g^{\prime}} \in \mathbf{m}$ for $g^{\prime} \prec_{t} g+f_{i}$.

The basis is said to be a cluster degree-triangular basis with respect to $t$, or a triangular basis for short, if it further contains the quantum cluster monomials in $t$ and $t[1]$.

It is not clear if a degree-triangular basis is unique or not. Nevertheless, a triangular basis must be unique if it exists, see [33, Lemma 6.3.2]. By definition, $\mathbf{I}^{t}$ is $\left(\prec_{t}, \mathbf{m}\right)$-unitriangular to the triangular basis.

We now propose the dual version below.

Definition 4.2 (codegree-triangular basis). A $\mathbb{k}$-basis $\mathbf{L}$ of $\mathrm{A}(t)$ is said to be a codegreetriangular basis with respect to $t$ if the following conditions hold:

(1) $X_{i}(t) \in \mathbf{L}$ for $i \in I$.

(2) Bar-invariance: $\mathbf{L}$ is invariant under the bar involution.

(3) Codegree parametrization: $\mathbf{L}$ is $M^{\circ}(t)$-copointed, i.e., it takes the form $\mathbf{L}=\left\{\mathbf{L}^{\eta} \mid \eta \in\right.$ $\left.M^{\circ}(t)\right\}$ such that $\mathbf{L}^{\eta}$ is $\eta$-copointed.

(4) Codegree triangularity: For any basis element $\mathbf{L}^{\eta}, i \in I$, the decomposition of the copointed function $\left\{\mathbf{L}^{\eta} * X_{i}(t)\right\}^{t}$ in terms of $\mathbf{L}$ is codegree $\left(\succ_{t}, \mathbf{m}\right)$-unitriangular:

$$
\left\{\mathbf{L}^{\eta} * X_{i}(t)\right\}^{t}=\sum_{\eta^{\prime} \succeq t \eta+f_{i}} c_{\eta^{\prime}} \mathbf{L}^{\eta^{\prime}}
$$

where $c_{\eta+f_{i}}=1, c_{\eta^{\prime}} \in \mathbf{m}$ for $\eta^{\prime} \succ_{t} \eta+f_{i}$.

The basis is said to be a cluster codegree-triangular basis with respect to $t$ if it further contains the quantum cluster monomials in $t$ and $t[-1]$.

By definition, $\mathbf{P}^{t}$ is codegree $\left(\succ_{t}, \mathbf{m}\right)$-unitriangular to the cluster codegree-triangular basis. Similar to [33, Lemma 6.3.2], we can show that the cluster codegree-triangular basis is unique if it exists.

Lemma 4.3 (factorization).

(1) Let there be given a degree-triangular basis $\mathbf{L}$. Then $\left[X_{i}(t) * S\right]^{t}=\left[S * X_{i}(t)\right]^{t} \in \mathbf{L}$ for any $i \in I_{\mathrm{f}}, S \in \mathbf{L}$ [33, Lemma 6.2.1].

(2) Let there be given a codegree-triangular basis $\mathbf{L}$. Then $\left\{X_{i}(t) * S\right\}^{t}=\left\{S * X_{i}(t)\right\}^{t} \in \mathbf{L}$ for any $i \in I_{\mathrm{f}}, S \in \mathbf{L}$. 
Definition 4.4 (bidegree-triangular basis). If $\mathrm{L}$ is both degree-triangular and codegree-triangular with respect to $t$, we call it a bidegree-triangular basis with respect to $t$.

Definition 4.5 (double triangular basis). If $\mathbf{L}$ is bidegree-triangular with respect to $t$ and further contains the quantum cluster monomials in $t, t[-1], t[1]$, we call it a cluster bidegree-triangular basis of $\mathrm{A}(t)$ or a double triangular basis with respect to $t$.

The basis $\mathbf{L}$ in Example 3.8 provides an example of double triangular bases.

Definition 4.6 (common triangular basis). Assume that $\mathbf{L}$ is the triangular basis of $\mathrm{A}(t)$ with respect to $t$. If, for any $t^{\prime} \in \Delta^{+}, \overleftarrow{\mu}_{t, t^{\prime}}^{*} \mathbf{L}$ is the triangular basis of $\mathrm{A}\left(t^{\prime}\right)=\overleftarrow{\mu}_{t, t^{\prime}}^{*} \mathrm{~A}(t)$ with respect to $t^{\prime}$ and is compatible with $\mathbf{L}$, we call $\mathbf{L}$ the common triangular basis.

\subsection{From triangular bases to double triangular bases}

Proposition 4.7. Let there be given the triangular basis $\mathbf{L}^{t}$ of $\mathrm{A}(t)$ with respect to the seed $t$. If $\mathbf{L}^{t[-1]}:=\overleftarrow{\mu}_{t, t[-1]}^{*} \mathbf{L}^{t}$ is the triangular basis with respect to $t[-1]$, then $\mathbf{L}^{t}$ is the double triangular basis with respect to $t$.

Proof. By assumption, $\mathbf{L}^{t}$ is the triangular basis for $t$ and $t[-1]$, thus it must contain the quantum cluster monomials in $t, t[1]$ and $t[-1], t$ respectively. It remains to check that $\mathbf{L}^{t}$ satisfies the defining conditions of a codegree triangular basis for $t$, see Definition 4.2.

Conditions (1) and (2) are trivial as $\mathbf{L}^{t}$ is assumed to be triangular for $t$. Since $\mathbf{L}^{t[-1]}$ is $M^{\circ}(t[-1])$-pointed, $\mathbf{L}^{t}=\overleftarrow{\mu}_{t[-1], t}^{*} \mathbf{L}^{t[-1]}$ is $M^{\circ}(t)$-copointed by Proposition 3.12. Thus Condition (3) is also verified.

Now, let us prove Condition (4).

First, consider any $i \in I_{\mathrm{f}}$. Then for any $V \in \mathbf{L}^{t}$ which is bipointed by (i), we have $\{V *$ $\left.X_{i}(t)\right\}^{t}=\left[V * X_{i}(t)\right]^{t}=\left[X_{i}(t) * V\right]^{t} \in \mathbf{L}^{t}$ by Lemma 4.3.

Second, consider any $k \in I_{\mathrm{uf}}$ and any $\eta$-copointed element $V \in \mathbf{L}^{t}$. Then $\overleftarrow{\mu}_{t, t[-1]}^{*} X_{k}(t)=$ $I_{\sigma^{-1} k}(t[-1])$, and $\overleftarrow{\mu}_{t, t[-1]}^{*} V$ is pointed at $g=\psi_{t[-1], t} \eta$. Since $\overleftarrow{\mu}_{t, t[-1]}^{*} V$ belongs to the triangular basis $\overleftarrow{\mu}_{t, t[-1]}^{*} \mathbf{L}^{t}=\mathbf{L}^{t[-1]}$, the normalized product

$$
Z:=\left[\overleftarrow{\mu}_{t, t[-1]}^{*} V * I_{\sigma^{-1} k}(t[-1])\right]^{t[-1]}=v^{\alpha} \overleftarrow{\mu}_{t, t[-1]}^{*} V * I_{\sigma^{-1} k}(t[-1])
$$

$\alpha \in \mathbb{Z}$, is $\left(\prec_{t[-1]}, \mathbf{m}\right)$-unitriangular to $\mathbf{I}^{t[-1]}$ by Lemma 3.21. Therefore, it is $\left(\prec_{t[-1]}, \mathbf{m}\right)$-unitriangular to $\mathbf{L}^{t[-1]}$. Then it has the following finite $\left(\prec_{t[-1]}, \mathbf{m}\right)$-unitriangular decomposition in $\mathbf{L}^{t[-1]}$ :

$$
Z=S^{(0)}+\sum_{j=1}^{r} b^{(j)} S^{(j)}
$$

with $b^{(j)} \in \mathbf{m}, r \in \mathbb{N}, \operatorname{deg}^{t[-1]} S^{(j)} \prec_{t[-1]} \operatorname{deg}^{t[-1]} S^{(0)}=\operatorname{deg}^{t[-1]} Z$ for $j>0$.

Applying the mutation $\overleftarrow{\mu}_{t[-1], t}^{*}$, we obtain

$$
Z^{\prime}:=\overleftarrow{\mu}_{t[-1], t}^{*} Z=v^{\alpha} V * X_{k}(t)=\overleftarrow{\mu}_{t[-1], t}^{*} S^{(0)}+\sum_{j=1}^{r} b^{(j)} \overleftarrow{\mu}_{t[-1], t}^{*} S^{(j)}
$$

Proposition 3.12 implies that $Z^{\prime}$ is copointed and, for any $j>0$, we must have

$$
\operatorname{codeg}^{t} \overleftarrow{\mu}_{t[-1], t}^{*} S^{(j)} \succ_{t} \operatorname{codeg}^{t} \overleftarrow{\mu}_{t[-1], t}^{*} S^{(0)}=\operatorname{codeg}^{t} Z^{\prime}
$$

Then this is a codegree $\left(\succ_{t}, \mathbf{m}\right)$-unitriangular decomposition in terms of the copointed set $\mathbf{L}^{t}$. 
We prove the following inverse result, although it will not be used in this paper.

Proposition 4.8. Assume that $\mathbf{L}^{t}$ is the double triangular basis of $\mathrm{A}(t)$ with respect to the seed $t$. Then $\mathbf{L}^{t[-1]}:=\overleftarrow{\mu}_{t, t[-1]}^{*} \mathbf{L}^{t}$ is the triangular basis with respect to $t[-1]$.

Proof. By assumption, $\mathbf{L}^{t[-1]}$ contains the quantum cluster monomials in $t[-1], t$. It remains to check that $\mathbf{L}^{t[-1]}$ satisfies the definition condition of a degree triangular basis for $t[-1]$.

(i) Since $\mathbf{L}^{t}$ is $M^{\circ}(t)$-copointed, $\mathbf{L}^{t[-1]}=\overleftarrow{\mu}_{t, t[-1]}^{*} \mathbf{L}^{t}$ is $M^{\circ}(t[-1])$-pointed by Proposition 3.12.

(ii-a) Take any $i \in I_{\mathrm{f}}$. Then for any $\left(g-f_{i}\right)$-pointed element $V \in \mathbf{L}^{t[-1]}$, we have $X_{i} * V=$ $v^{\alpha} X_{i} \cdot V=v^{2 \alpha} V * X_{i}$ for some $\alpha \in \mathbb{Z}$. Since $X_{i} \cdot V$ is $g$-pointed, it agrees with $\left[X_{i} * V\right]^{t[-1]}$. Moreover, $\overleftarrow{\mu}_{t[-1], t}^{*}\left(X_{i} \cdot V\right)$ is $\eta$-copointed by Proposition 3.12, where $\eta=\psi_{t[-1], t}^{-1} g$. Therefore, $\overleftarrow{\mu}_{t[-1], t}^{*}\left(v^{-\alpha} X_{i} * V\right)=v^{-\alpha} X_{i} * \overleftarrow{\mu}_{t[-1], t}^{*} V$ agrees with the copointed function $\left\{X_{i} * \overleftarrow{\mu}_{t[-1], t}^{*} V\right\}^{t}$ Using Lemma 4.3, we deduce that $\overleftarrow{\mu}_{t[-1], t}^{*}\left[X_{i} * V\right]^{t[-1]}=\left\{X_{i} * \overleftarrow{\mu}_{t[-1], t}^{*} V\right\}^{t}$ is contained in the codegree triangular basis $\mathbf{L}^{t}$. Consequently, $\left[X_{i} * V\right]^{t[-1]}$ belongs to $\mathbf{L}^{t[-1]}$.

(ii-b) Take any $k \in I_{\text {uf }}$ and $g$-pointed element $V \in \mathbf{L}^{t[-1]}$. Then $\overleftarrow{\mu}_{t[-1], t}^{*} X_{k}(t[-1])=$ $P_{\sigma k}(t) \in \mathbf{L}^{t}$, and $\overleftarrow{\mu}_{t[-1], t}^{*} V$ is copointed at $\eta=\psi_{t[-1], t}^{-1} g$. The function $\overleftarrow{\mu}_{t[-1], t}^{*}\left[X_{k}(t[-1]) * V\right]^{t[-1]}$ is copointed by Proposition 3.12, i.e., $\overleftarrow{\mu}_{t[-1], t}^{*}\left[X_{k}(t[-1]) * V\right]^{t[-1]}=\left\{P_{\sigma k}(t) * \overleftarrow{\mu}_{t[-1], t}^{*} V\right\}^{t}$. Since $\mathbf{L}^{t}$ is a double triangular basis, $\overleftarrow{\mu}_{t[-1], t}^{*} V$ is codegree $\left(\succ_{t}, \mathbf{m}\right)$-unitriangular to $\mathbf{P}^{t}$. Lemma $3.21 \mathrm{im}-$ plies that $\left\{P_{\sigma k}(t) * \overleftarrow{\mu}_{t[-1], t}^{*} V\right\}^{t}$ is codegree $\left(\succ_{t}, \mathbf{m}\right)$-unitriangular to $\mathbf{P}^{t}$ and, consequently, is codegree $\left(\succ_{t}, \mathbf{m}\right)$-unitriangular to $\mathbf{L}^{t}$. We obtain a finite codegree $\left(\succ_{t}, \mathbf{m}\right)$-unitriangular decomposition

$$
Z:=\left\{P_{\sigma k}(t) * \overleftarrow{\mu}_{t[-1], t}^{*} V\right\}^{t}=\sum_{j=0}^{r-1} b^{(j)} S^{(j)}+S^{(r)}
$$

with $r \in \mathbb{N}, b^{(j)} \in \mathbf{m}$, codeg $S^{(j)} \succ_{t} \operatorname{codeg}^{t} S^{(r)}=\operatorname{codeg}^{t} Z$ for $j<r$.

Applying the mutation $\overleftarrow{\mu}_{t, t[-1]}^{*}$, we obtain

$$
Z^{\prime}:=\overleftarrow{\mu}_{t, t[-1]}^{*} Z=\left[X_{k}(t[-1]) * V\right]^{t[-1]}=\sum_{j=1}^{r} b^{(j)} \overleftarrow{\mu}_{t, t[-1]}^{*} S^{(j)}+\overleftarrow{\mu}_{t, t[-1]}^{*} S^{(r)}
$$

Proposition 3.12 implies that $Z^{\prime}$ is pointed and, for any $j<r$, we have $\operatorname{deg}^{t[-1]} \overleftarrow{\mu}_{t, t[-1]}^{*} S^{(j)} \prec_{t[-1]}$ $\operatorname{deg}^{t[-1]} \overleftarrow{\mu}_{t, t[-1]}^{*} S^{(r)}=\operatorname{deg}^{t[-1]} Z^{\prime}$. Therefore, this decomposition becomes a degree $\left(\prec_{t[-1]}, \mathbf{m}\right)-$ unitriangular decomposition in $\mathbf{L}^{t}$.

\subsection{Properties of common triangular bases}

Recall that we have the $\mathbb{k}$-algebra anti-isomorphism $\iota: \mathcal{L P}(t) \simeq \mathcal{L P}\left(t^{\text {op }}\right)$ such that $\iota\left(X^{m}\right)=X^{m}$, see (3.1). Define the subalgebra $\mathrm{A}\left(t^{\mathrm{op}}\right)=\iota \mathrm{A}(t) \subset \mathcal{U}_{q}\left(t^{\mathrm{op}}\right)$.

Proposition 4.9. If $\mathrm{A}(t)$ possesses the common triangular basis $\mathbf{L} \subset \mathcal{L P}(t)$, then $\mathrm{A}\left(t^{\mathrm{op}}\right)$ possesses the common triangular basis $\iota \mathbf{L} \subset \mathcal{L} \mathcal{P}\left(t^{\mathrm{op}}\right)$.

Proof. Notice that $\iota$ sends (quantum) cluster monomials $\overleftarrow{\mu}_{t^{\prime}, t}^{*} X\left(t^{\prime}\right)^{m}$ to (quantum) cluster monomials $\overleftarrow{\mu}_{\left(t^{\prime}\right)^{\mathrm{op}, t_{\mathrm{op}}}}^{*} X\left(\left(t^{\prime}\right)^{\mathrm{op}}\right)^{m}, m \in \mathbb{N}^{I_{\mathrm{uf}}}$, because it commutes with mutations. In particular, it gives a bijection between the sets of cluster monomials.

For any seed $t^{\prime} \in \Delta^{+}$, because the common triangular basis $\mathbf{L}$ gives rise to the double triangular basis for $t^{\prime}$ by Proposition 4.7, it gives rise to a codegree triangular basis $\mathbf{L}^{t^{\prime}} \subset \mathcal{L} \mathcal{P}\left(t^{\prime}\right)$ for $t^{\prime}$. Then $\iota \mathbf{L}^{t^{\prime}} \subset \mathcal{L} \mathcal{P}\left(\left(t^{\prime}\right)^{\mathrm{op}}\right)$ is a degree triangular basis containing all cluster monomials. Therefore, $\iota \mathbf{L}^{t^{\prime}}$ is the triangular basis with respect to $\left(t^{\prime}\right)^{\mathrm{op}}$. 
Moreover, for any $t, t^{\prime} \in \Delta^{+}$, because the elements of $\mathbf{L}$ are compatibly pointed at $t[-1]$, $t^{\prime}[-1]$, the elements of $\mathbf{L}$ are compatibly copointed at $t, t^{\prime}$ by Proposition 3.23. It follows that the elements of $\iota \mathbf{L}$ are compatibly pointed at $t^{\mathrm{op}},\left(t^{\prime}\right)^{\mathrm{op}}$.

Therefore, $\iota \mathbf{L}$ is the common triangular basis by definition.

Recall that a common triangular basis is necessarily compatibly pointed at $\Delta^{+}$. We have the following results.

Theorem 4.10. Let there be a $\mathbb{k}$-subalgebra $\mathrm{A}(t)$ of the upper quantum cluster algebra $\mathcal{U}_{q}(t)$. Assume that $\mathrm{A}(t)$ possesses the common triangular basis $\mathbf{L}$. Then the following statements are true.

(1) $\overleftarrow{\mu}_{t, t^{\prime}}^{*} \mathbf{L}$ is the double triangular basis of $\mathrm{A}\left(t^{\prime}\right)=\overleftarrow{\mu}_{t, t^{\prime}}^{*} \mathrm{~A}(t)$ for any seed $t^{\prime} \in \Delta^{+}$

(2) $\mathbf{L}$ is compatibly copointed at $\Delta^{+}$.

Proof. (1) The claim follows from Proposition 4.7. (2) By Proposition 4.9, $\iota \mathbf{L}$ is the common triangular basis of $\mathrm{A}\left(t^{\mathrm{op}}\right)$, which is necessarily compatibly pointed at $\left(\Delta^{+}\right)^{\mathrm{op}}$. Applying $\iota$ again, we deduce that $\mathbf{L}=\iota(\iota \mathbf{L})$ is compatibly copointed at $\Delta^{+}$.

\section{Main results}

\subsection{An analog of Leclerc's conjecture}

Let there be given an injective-reachable seed $t$ and a $\mathbb{k}$-subalgebra $\mathrm{A}(t)$ of the upper quantum cluster algebra $\mathcal{U}_{q}(t)$.

Lemma 5.1. Assume that $\mathrm{A}(t)$ possesses a bidegree-triangular basis $\mathbf{L}$. Take any $i \in I$ and $\gamma \in M^{\circ}(t)$. Denote the codegree of the $\gamma$-pointed basis element $\mathbf{L}_{\gamma}$ by $\eta$. Then we have either $X_{i}(t) * \mathbf{L}_{\gamma} \in v^{\mathbb{Z}} \mathbf{L}$ or

$$
X_{i}(t) * \mathbf{L}_{\gamma}=v^{s} S+\sum_{j} b_{j} L^{(j)}+v^{h} H
$$

such that $s>h \in \mathbb{Z}, b_{j} \in v^{h+1} \mathbb{Z}[v] \cap v^{s-1} \mathbb{Z}\left[v^{-1}\right]$, and $S, L^{(j)}, H$ are finitely many distinct elements of $\mathbf{L}$ with

$$
\begin{aligned}
& \operatorname{deg}^{t} H, \operatorname{deg}^{t} L^{(j)} \prec_{t} \operatorname{deg}^{t} S=f_{i}+\gamma, \\
& \operatorname{codeg}^{t} S, \operatorname{codeg}^{t} L^{(j)} \succ_{t} \operatorname{codeg}^{t} H=f_{i}+\eta .
\end{aligned}
$$

Moreover, we have $s=\lambda\left(f_{i}, \gamma\right), h=\lambda\left(f_{i}, \eta\right)$.

Proof. Omit the symbol $t$ for simplicity.

Denote the codegree of $\mathbf{L}_{\gamma}$ by $\eta=\gamma+\widetilde{B} n$, where $n \in N_{\text {uf }}^{\geq 0}(t) \simeq \mathbb{N}^{I_{\text {uf }}}$. Then $X_{i} * \mathbf{L}_{\gamma}$ has degree $f_{i}+\gamma$ with coefficient $v^{s}:=v^{\lambda\left(f_{i}, \gamma\right)}$, codegree $f_{i}+\eta$ with coefficient $v^{h}:=v^{\lambda\left(f_{i}, \eta\right)}$. It follows that $h=s+\lambda\left(f_{i}, \widetilde{B} n\right) \leq s$, where $h=s$ if and only if $n_{i}=0$.

Because $\mathbf{L}$ is a degree-triangular basis, we have a degree $\left(\prec_{t}, \mathbf{m}\right)$-unitriangular decomposition with finitely many $S^{(0)}, \ldots, S^{(r)} \in \mathbf{L}$ :

$$
\left[X_{i} * \mathbf{L}_{\gamma}\right]^{t}=v^{-s} X_{i} * \mathbf{L}_{\gamma}=S^{(0)}+\sum_{j>0} b^{(j)} S^{(j)}
$$

such that $b^{(j)} \in \mathbf{m}, \operatorname{deg} S^{(j)} \prec \operatorname{deg} S^{(0)}=f_{i}+\gamma$ for $j>0$.

(i) Assume $n_{i}=0$, then $v^{-s} X_{i} * \mathbf{L}_{\gamma}$ is pointed and bar-invariant. Because every basis elements $S^{(j)}$ appearing in (5.1) are bar-invariant and $b^{(j)} \in \mathbf{m}$, it follows that $v^{-s} X_{i} * \mathbf{L}_{\gamma}=$ $S^{(0)} \in \mathbf{L}$. 
(ii) Assume $n_{i} \neq 0$. Then $h<s$. In addition, $v^{-s} X_{i} * \mathbf{L}_{\gamma}$ is pointed but not bar-invariant, because it has the Laurent monomial $v^{h-s} X^{\eta+f_{i}}$ at the codegree.

Notice that $v^{-h} X_{i} * \mathbf{L}_{\gamma}$ is copointed. Multiplying the decomposition (5.1) by $v^{s-h}$ and applying the bar involution, we get a decomposition of copointed elements

$$
v^{h} \mathbf{L}_{\gamma} * X_{i}=v^{h-s} S^{(0)}+\sum_{j>0} v^{h-s} \cdot \overline{b^{(j)}} S^{(j)} .
$$

Because $\mathbf{L}$ is a codegree-triangular basis and $v^{h} \mathbf{L}_{\gamma} * X_{i}$ is copointed, the above decomposition must be codegree $\left(\succ_{t}, \mathbf{m}\right)$-unitriangular. But $v^{h-s} S^{(0)}$ is not copointed since $S^{(0)} \in \mathbf{L}$ is copointed but $h<s$. Relabeling $S^{(j)}, j>0$, if necessary, we assume $\operatorname{codeg} S^{(j)} \succ_{t} \operatorname{codeg} S^{(r)}$ for $j<r$. Then the codegree term $X^{\eta+f_{i}}$ is contributed from $S^{(r)}$ and $S^{(r)}$ is copointed at $\operatorname{codeg}\left(\mathbf{L}_{\gamma} * X_{i}\right)=\eta+f_{i}$ with decomposition coefficient $1=v^{h-s} \overline{b^{(r)}}$. In addition, the remaining terms $S^{(j)}, 0<j<r$ must have coefficients $v^{h-s} \cdot \overline{b^{(j)}}$ in $\mathbf{m}$. It follows that $b_{j}:=b^{(j)} v^{s}$ belongs to $v^{h+1} \mathbb{Z}[v]$ for $0<j<r$. The claim follows by taking $S=S^{(0)}, H=S^{(r)}, L^{(j)}=S^{(j)}$ for $0<j<r$.

Theorem 5.2. Let there be given a $\mathbb{k}$-subalgebra $\mathrm{A}(t)$ of the upper quantum cluster algebra $\mathcal{U}_{q}(t)$. Assume that it has the common triangular basis $\mathbf{L}$. Then, for any $i \in I, V \in \mathbf{L}$, and any localized quantum cluster monomial $R$, we have either $R * V \in v^{\mathbb{Z}} \mathbf{L}$ or

$$
R * V=v^{s} S+\sum_{j} b_{j} L^{(j)}+v^{h} H
$$

such that $s>h \in \mathbb{Z}, b_{j} \in v^{h+1} \mathbb{Z}[v] \cap v^{s-1} \mathbb{Z}\left[v^{-1}\right]$, and $S, L^{(j)}, H$ are finitely many distinct elements of $\mathbf{L}$.

Proof. Since $\mathbf{L}$ is the common triangular basis, Theorem 4.10(1) implies that $\overleftarrow{\mu}_{t, t^{\prime}}^{*} \mathbf{L}$ is the double triangular basis (and thus bidegree-triangular) of $\mathrm{A}\left(t^{\prime}\right)=\overleftarrow{\mu}_{t, t^{\prime}}^{*} \mathrm{~A}(t)$ for any seed $t^{\prime} \in \Delta^{+}$. We apply Lemma 5.1 for localized quantum cluster monomials associated to $t^{\prime}$.

Theorem 5.2 is a weaker form of the following analog of Leclerc's conjecture.

Conjecture 5.3. Assume that $\mathbf{L}$ is the common triangular basis. Assume that $R$ is a real basis element in $\mathbf{L}$ (i.e., $R^{2} \in \mathbf{L}$ ). Then, for any $V \in \mathbf{L}$, we have either $R * V \in v^{\mathbb{Z}} \mathbf{L}$ or

$$
R * V=v^{s} S+\sum_{j} b_{j} L^{(j)}+v^{h} H
$$

such that $s>h \in \mathbb{Z}, b_{j} \in v^{h+1} \mathbb{Z}[v] \cap v^{s-1} \mathbb{Z}\left[v^{-1}\right]$, and $S, L^{(j)}, H$ are finitely many distinct elements of $\mathbf{L}$.

Choose any $l \in \mathbb{N}$. Let $\mathcal{C}_{l}$ denote a level- $l$ subcategory of the monoidal category of the finite-dimensional modules of a quantum affine algebra $\mathrm{U}_{\mathrm{q}}(\widehat{\mathfrak{g}})$ in the sense of [16], where $\mathfrak{g}$ is a Lie algebra of type $A D E$. Let $K_{t}\left(\mathcal{C}_{l}\right)$ denote its $t$-deformed Grothendieck ring, $t$ a quantum parameter. By [33, Theorem 8.4.3], $K_{t}\left(\mathcal{C}_{l}\right)$ is a (partially compactified) quantum cluster algebra $\overline{\mathcal{A}}_{q}$. Notice that $K_{t}\left(\mathcal{C}_{l}\right)$ has a bar-invariant basis $\{[S]\}$, where $S$ are simple modules. By [33], $\{[S]\}$ becomes the common triangular basis of the corresponding quantum cluster algebra $\mathcal{A}_{q}$ after localization at the frozen factors.

A simple module $R$ in $\mathcal{C}_{l}$ is called real if $R \otimes R$ remains simple. Theorem 5.2 implies the following result. 
Theorem 5.4. Let $R$ be any real simple module in $\mathcal{C}_{l}$ corresponding to a cluster monomial. Then, for any simple modules $V \in \mathcal{C}_{l}$, either $R \otimes V$ is simple, or there exists finitely many distinct simple modules $S, L^{(j)}, H$ in $\mathcal{C}_{l}$ such that the following equation holds in the deformed Grothendieck ring $K_{t}\left(\mathcal{C}_{l}\right)$ :

$$
[R] *[V]=t^{s}[S]+\sum_{j} b_{j}\left[L^{(j)}\right]+t^{h}[H]
$$

where $s>h \in \frac{1}{2} \mathbb{Z}, b_{j} \in t^{h+\frac{1}{2}} \mathbb{Z}\left[t^{\frac{1}{2}}\right] \cap t^{s-\frac{1}{2}} \mathbb{Z}\left[t^{-\frac{1}{2}}\right]$.

Notice that we can replace $[S]$ by the $t$-analog of $q$-character of $S$ and embed $K_{t}\left(\mathcal{C}_{l}\right)$ into the completion of a quantum torus, see [15, 31, 38]. Correspondingly, Theorem 5.4 gives an algebraic relation for such characters.

Remark 5.5. Assume that the quantum cluster algebra arises from a quantum unipotent subgroup of symmetric Kac-Moody type, which possesses the dual canonical basis corresponding to the set of self-dual simple modules of the corresponding quiver Hecke algebra. In this case, up to $v$-power rescaling, $S$ and $H$ correspond to the simple socle and simple head of the convolution product $R \circ V$ respectively. See [17, Section 4] for more details.

Similar picture appears in the category of finite-dimensional modules of quantum affine algebras $\mathrm{U}_{\mathrm{q}}(\widehat{\mathfrak{g}})$ by [21], where the objects are not graded. In particular, [21, Corollary 4.12] implies that, as a stronger version of Theorem 5.4, Conjecture 5.3 holds true for the quantum cluster algebras $K_{t}\left(\mathcal{C}_{l}\right)$.

\subsection{Properties of dual canonical bases}

Let us consider the quantum unipotent subgroup $\mathrm{A}_{\mathrm{q}}\left[N_{-}(w)\right]$ of symmetrizable Kac-Moody types in the sense of $[25,35]$. It is isomorphic to a (partially compactified) quantum cluster algebra after rescaling, see $[11,12]$ or [35]. Theorem 5.2 implies the following weaker version of Conjecture 1.1.

Theorem 5.6. Consider the dual canonical basis $\mathrm{B}^{\mathrm{up}}(w)$ of $\mathrm{A}_{\mathrm{q}}\left[N_{-}(w)\right]$. If $b_{1} \in \mathrm{B}^{\mathrm{up}}(w)$ corresponds to a quantum cluster monomial after rescaling, then for any $b_{2} \in \mathrm{B}^{\mathrm{up}}(w)$, either $b_{1} b_{2} \in q^{\mathbb{Z}} \mathrm{B}^{\mathrm{up}}(w)$ or $(1.1)$ holds true.

Proof. By [35, Theorem 9.5.1], after rescaling and localization at the frozen factors, the dual canonical basis $\mathrm{B}^{\mathrm{up}}(w)$ of $\mathrm{A}_{\mathrm{q}}\left[N_{-}(w)\right]$ becomes the common triangular basis of the corresponding quantum cluster algebra. Therefore, elements of $\mathrm{B}^{\mathrm{up}}(w)$ satisfy the algebraic relation (5.2) after rescaling. Notice that the rescaling factors depends on the natural root-lattice grading of $\mathrm{U}_{\mathrm{q}}$, which is homogeneous for $\overleftarrow{\mu}_{t^{\prime}, t}^{*} X_{i}\left(t^{\prime}\right) * V, S, L^{(j)}, H$ in (5.2), because the $Y$-variables have 0 -grading [35, Section 9.1]. The claim follows from Theorem 5.2.

Theorem 5.6 would imply Conjecture 1.1 if the following multiplicative reachability conjecture can be proved.

Conjecture 5.7. If $b \in \mathrm{B}^{\mathrm{up}}(w) \subset \mathrm{A}_{\mathrm{q}}\left[N_{-}(w)\right]$ is real (i.e., $b^{2} \in q^{\mathbb{Z}} \mathrm{B}^{\mathrm{up}}(w)$ ), then it corresponds to a quantum cluster monomial after rescaling.

Conjecture 5.7 can be generalized as the following, which implies Conjecture 5.3 by Theorem 5.2.

Conjecture 5.8 (multiplicative reachability conjecture). Let $\mathbf{L}$ denote a common triangular basis. If $b \in \mathbf{L}$ is real (i.e., $\left.b^{2} \in \mathbf{L}\right)$, then it corresponds to a localized quantum cluster monomial. 
Remark 5.9 (reachability conjectures). When the cluster algebra admits an additive categorification by triangulated categories (cluster categories), we often expect that the rigid objects (objects with vanishing self-extensions) correspond to the (quantum) cluster monomials. If so, such objects can be constructed from the initial cluster tilting objects via (categorical) mutations. Let us call such an expectation the additive reachability conjecture. This conjecture is not true for a general cluster algebra because the cluster algebra seems too small for the cluster category.

When the cluster algebra admits a monoidal categorification by monoidal categories, we similarly expect that the real simple objects correspond to the (quantum) cluster monomials (see [16]). If so, such objects can be constructed from the an initial collection of real simple objects via (categorical) mutations. Let us call such an expectation the multiplicative reachability conjecture. Conjecture 5.7 is related to the special case for $\mathrm{A}_{\mathrm{q}}\left[N_{-}(w)\right]$.

We also conjecture an equivalence between the additive reachability conjecture and the multiplicative reachability conjecture, which can be viewed as an analog of the open orbit conjecture [9, Conjecture 18.1]. See [32, Section 1] for a comparison between additive categorification and monoidal categorification.

All these conjectures are largely open.

\section{Acknowledgements}

The author thanks the referees for helpful suggestions. He also thanks an anonymous referee for informing him of the work [21]. The author was supported by the National Natural Science Foundation of China (Grant No. 11701365).

\section{References}

[1] Berenstein A., Fomin S., Zelevinsky A., Cluster algebras. III. Upper bounds and double Bruhat cells, Duke Math. J. 126 (2005), 1-52, arXiv:math.RT/0305434.

[2] Berenstein A., Zelevinsky A., Quantum cluster algebras, Adv. Math. 195 (2005), 405-455, arXiv:math.QA/0404446.

[3] Cautis S., Williams H., Cluster theory of the coherent Satake category, J. Amer. Math. Soc. 32 (2019), 709-778, arXiv:1801.08111.

[4] Derksen H., Weyman J., Zelevinsky A., Quivers with potentials and their representations II: applications to cluster algebras, J. Amer. Math. Soc. 23 (2010), 749-790, arXiv:0904.0676.

[5] Fock V.V., Goncharov A.B., Moduli spaces of local systems and higher Teichmüller theory, Publ. Math. Inst. Hautes Études Sci. (2006), 1-211, arXiv:math.AG/0311149.

[6] Fock V.V., Goncharov A.B., Cluster ensembles, quantization and the dilogarithm, Ann. Sci. Éc. Norm. Supér. (4) 42 (2009), 865-930, arXiv:math.AG/0311245.

[7] Fomin S., Zelevinsky A., Cluster algebras. I. Foundations, J. Amer. Math. Soc. 15 (2002), 497-529, arXiv:math.RT/0104151.

[8] Fomin S., Zelevinsky A., Cluster algebras. IV. Coefficients, Compos. Math. 143 (2007), 112-164, arXiv:math.RA/0602259.

[9] Geiß C., Leclerc B., Schröer J., Kac-Moody groups and cluster algebras, Adv. Math. 228 (2011), 329-433, arXiv:1001.3545.

[10] Geiß C., Leclerc B., Schröer J., Cluster structures on quantum coordinate rings, Selecta Math. (N.S.) 19 (2013), 337-397, arXiv:1104.0531.

[11] Goodearl K., Yakimov M., Integral quantum cluster structures, arXiv:2003.04434.

[12] Goodearl K.R., Yakimov M.T., Quantum cluster algebra structures on quantum nilpotent algebras, Mem. Amer. Math. Soc. 247 (2017), vii+119 pages.

[13] Gross M., Hacking P., Keel S., Birational geometry of cluster algebras, Algebr. Geom. 2 (2015), 137-175, arXiv:1309.2573. 
[14] Gross M., Hacking P., Keel S., Kontsevich M., Canonical bases for cluster algebras, J. Amer. Math. Soc. 31 (2018), 497-608, arXiv:1411.1394.

[15] Hernandez D., Algebraic approach to q, t-characters, Adv. Math. 187 (2004), 1-52, arXiv:math.QA/0212257.

[16] Hernandez D., Leclerc B., Cluster algebras and quantum affine algebras, Duke Math. J. 154 (2010), 265-341, arXiv:0903.1452.

[17] Kang S.J., Kashiwara M., Kim M., Oh S.-J., Monoidal categorification of cluster algebras, J. Amer. Math. Soc. 31 (2018), 349-426, arXiv:1801.05145.

[18] Kashiwara M., Bases cristallines, C. R. Acad. Sci. Paris Sér. I Math. 311 (1990), 277-280.

[19] Kashiwara M., On crystal bases of the $Q$-analogue of universal enveloping algebras, Duke Math. J. 63 (1991), $465-516$.

[20] Kashiwara M., Kim M., Laurent phenomenon and simple modules of quiver Hecke algebras, Compos. Math. 155 (2019), 2263-2295, arXiv:1811.02237.

[21] Kashiwara M., Kim M., Oh S.-J., Park E., Monoidal categorification and quantum affine algebras, Compos. Math. 156 (2020), 1039-1077, arXiv:1910.08307.

[22] Keller B., Cluster algebras, quiver representations and triangulated categories, in Triangulated Categories, London Math. Soc. Lecture Note Ser., Vol. 375, Cambridge Univetsity Press, Cambridge, 2010, 76-160, arXiv:0807.1960.

[23] Khovanov M., Lauda A.D., A diagrammatic approach to categorification of quantum groups. I, Represent. Theory 13 (2009), 309-347, arXiv:0803.4121.

[24] Khovanov M., Lauda A.D., A categorification of quantum sl(n), Quantum Topol. 1 (2010), 1-92, arXiv:0807.3250.

[25] Kimura Y., Quantum unipotent subgroup and dual canonical basis, Kyoto J. Math. 52 (2012), 277-331, arXiv:1010.4242.

[26] Kimura Y., Qin F., Graded quiver varieties, quantum cluster algebras and dual canonical basis, Adv. Math. 262 (2014), 261-312, arXiv:1205.2066.

[27] Leclerc B., Imaginary vectors in the dual canonical basis of $U_{q}(\mathfrak{n})$, Transform. Groups 8 (2003), 95-104, arXiv:math.QA/0202148.

[28] Lusztig G., Canonical bases arising from quantized enveloping algebras, J. Amer. Math. Soc. 3 (1990), 447-498.

[29] Lusztig G., Quivers, perverse sheaves, and quantized enveloping algebras, J. Amer. Math. Soc. 4 (1991), 365-421.

[30] Lusztig G., Total positivity in reductive groups, in Lie Theory and Geometry, Progr. Math., Vol. 123, Birkhäuser Boston, Boston, MA, 1994, 531-568.

[31] Nakajima H., Quiver varieties and $t$-analogs of $q$-characters of quantum affine algebras, Ann. of Math. 160 (2004), 1057-1097, arXiv:math.QA/0105173.

[32] Nakajima H., Quiver varieties and cluster algebras, Kyoto J. Math. 51 (2011), 71-126, arXiv:0905.0002.

[33] Qin F., Triangular bases in quantum cluster algebras and monoidal categorification conjectures, Duke Math. J. 166 (2017), 2337-2442, arXiv:1501.04085.

[34] Qin F., Bases for upper cluster algebras and tropical points, arXiv:1902.09507.

[35] Qin F., Dual canonical bases and quantum cluster algebras, arXiv:2003.13674.

[36] Rouquier R., 2-Kac-Moody algebras, arXiv:0812.5023.

[37] Tran T., F-polynomials in quantum cluster algebras, Algebr. Represent. Theory 14 (2011), 1025-1061, arXiv:0904.3291.

[38] Varagnolo M., Vasserot E., Perverse sheaves and quantum Grothendieck rings, in Studies in Memory of Issai Schur (Chevaleret/Rehovot, 2000), Progr. Math., Vol. 210, Birkhäuser Boston, Boston, MA, 2003, 345-365, arXiv:math.QA/0103182. 